\title{
Oxygen-deficient perovskites for oxygen evolution reaction in alkaline media: a review
}

\author{
Ahmed Badreldin ${ }^{1} \cdot$ Aya E. Abusrafa $^{1} \cdot$ Ahmed Abdel-Wahab $^{1}$ (I) \\ Received: 10 August 2020 / Accepted: 3 September 2020 / Published online: 21 September 2020 \\ (C) The Author(s) 2020
}

\begin{abstract}
Oxygen vacancies in complex metal oxides and specifically in perovskites are demonstrated to significantly enhance their electrocatalytic activities due to facilitating a degree of control in the material's intrinsic properties. The reported enhancement in intrinsic OER activity of oxygen-deficient perovskites surfaces has inspired their fabrication via a myriad of schemes. Oxygen vacancies in perovskites are amongst the most favorable anionic or Schottky defects to be induced due to their low formation energies. This review discusses recent efforts for inducing oxygen vacancies in a multitude of perovskites, including facile and environmentally benign synthesis strategies, characterization techniques, and detailed insight into the intrinsic mechanistic modulation of perovskite electrocatalysts. Experimental, analytical, and computational techniques dedicated to the understanding of the improvement of OER activities upon oxygen vacancy induction are summarized in this work. The identification and utilization of intrinsic activity descriptors for the modulation of configurational structure, improvement in bulk charge transport, and favorable inflection of the electronic structure are also discussed. It is our foresight that the approaches, challenges, and prospects discussed herein will aid researchers in rationally designing highly active and stable perovskites that can outperform noble metal-based OER electrocatalysts.
\end{abstract}

\section{Introduction}

The global energy crisis associated with the increasing demand for energy as well as the rapid depletion of fossil fuels and carbon dioxide $\left(\mathrm{CO}_{2}\right)$ emissions associated with their use is one of the grand challenges facing humanity today. In fact, the International Energy Agency reported that the global energy demand is expected to rise to 26 TW by 2040 compared with $18 \mathrm{TW}$ in 2013, corresponding to $44 \mathrm{Gt} /$ year of $\mathrm{CO}_{2}$ emissions [1]. This has motivated the global community to maximize deployment of renewable energy sources in order to meet the ever-growing energy demand in a sustainable manner $[2,3]$. Hydrogen $\left(\mathrm{H}_{2}\right)$ being a carbon-free energy carrier is an ideal sustainable energy source that can potentially replace non-renewable carbon-based fossil fuels due to its high specific energy [4-6]. Currently, the primary technologies that are used to produce hydrogen include partial oxidation, steam reforming of methane, and coal gasification.

Ahmed Abdel-Wahab

ahmed.wahab@qatar.tamu.edu

1 Chemical Engineering Program, Texas A\&M University at Qatar, P.O. Box 23874, Doha, Qatar
However, these technologies involve the generation of significant amounts of greenhouse gasses, which is the main cause for global warming $[7,8]$.

Amongst various progressive technologies, electrochemical water splitting driven by electricity produced from renewable energy sources, such as solar or wind energy, has emerged as an effective approach to sustainably produce high-purity hydrogen with zero carbon emissions [1,9]. Photochemical and photoelectrochemical water splitting materials have also seen an afresh interest in research over the past decade. However, they face limitations due to reactordesign predicaments $[10,11]$. Water splitting is composed of two half-reactions, including the hydrogen evolution reaction (HER), at the cathode $\left(2 \mathrm{H}^{+}(\mathrm{aq})+2 \mathrm{e}^{-} \rightarrow \mathrm{H}_{2}\right.$ (g) acidic, $2 \mathrm{H}_{2} \mathrm{O}+2 \mathrm{e}^{-} \rightarrow \mathrm{H}_{2}+2 \mathrm{OH}^{-}$alkaline) and the oxygen evolution reaction $(\mathrm{OER})$ at the anode $\left(\mathrm{H}_{2} \mathrm{O}(\mathrm{l}) \rightarrow 1 / 2 \mathrm{O}_{2}(\mathrm{~g})+2 \mathrm{H}^{+}\right.$(aq) + $2 \mathrm{e}^{-}$acidic, $2 \mathrm{OH}^{-} \rightarrow 1 / 2 \mathrm{O}_{2}+\mathrm{H}_{2} \mathrm{O}+2 \mathrm{e}^{-}$alkaline) [7]. Under standard conditions, water-splitting electrolyzers require a predicted thermodynamic potential of $1.23 \mathrm{~V}$ to drive the HER and OER half-reactions in alkaline and acidic media [12]. However, water splitting is kinetically an uphill reaction and entails sluggish reaction kinetics especially for the OER. This inflicts a considerable overpotential requirement (the difference between the thermodynamic potential and 
experimentally measured potential) to overcome the kinetic energy barriers and generate appreciable current densities [2]. Practically, the applied potentials required to drive water splitting processes are around 1.8-2.2 V, which consumes approximately $50 \%$ excess potential than the thermodynamic equilibrium value. The latter results in low energy conversion efficiencies which hampered the widespread application of water-splitting technology.

Achieving high electrocatalytic activities hence improved conversion efficiencies in water-splitting electrolyzers that can be realized by determining the origin of overpotential through fundamentally understanding the correlation between the electrocatalytic activity and the water-splitting reactions mechanism. The OER is the limiting reaction in water splitting and requires high overpotential because it involves 4-electron transfer steps [13, 14]. An additional kinetic energy barrier is involved with every reaction step where the slowest step is regarded as the rate-determining step (RDS), and it can impose a significant increase in the overpotential required to drive the overall water-splitting reaction.

Alkaline OER proceeds via the oxidation of an adsorbed hydroxyl anion on the metal active site (M) to form an $\mathrm{M}-\mathrm{OH}$ intermediate (Eq. (1)). Then, protons and electrons are removed from M-OH to form M-O (Eq. (2)). Subsequently, oxygen formation follows two possible pathways. In one route, $\mathrm{M}-\mathrm{O}$ reacts with a hydroxyl anion to form hydroperoxide $\mathrm{M}-\mathrm{OOH}$ followed by another proton-coupled electron transfer process to produce molecular $\mathrm{O}_{2}$ (Eqs. (3) and (4)) [15-18]. In another route, a direct combination of two M-O intermediates produces $\mathrm{O}_{2}$ (Eq. (5)) [19].

$$
\begin{aligned}
& \mathrm{OH}^{-} \rightarrow \mathrm{M}-\mathrm{OH}+\mathrm{e}^{-} \\
& \mathrm{M}-\mathrm{OH}+\mathrm{OH}^{-} \rightarrow \mathrm{M}-\mathrm{O}+\mathrm{H}_{2} \mathrm{O}+\mathrm{e}^{-} \\
& \mathrm{M}-\mathrm{O}+\mathrm{OH}^{-} \rightarrow \mathrm{M}-\mathrm{OOH}+\mathrm{e}^{-} \\
& \mathrm{M}-\mathrm{OOH}+\mathrm{OH}^{-} \rightarrow \mathrm{O}_{2}+\mathrm{H}_{2} \mathrm{O}+\mathrm{e}^{-} \\
& \mathrm{M}-\mathrm{O}+\mathrm{M}-\mathrm{O} \rightarrow \mathrm{O}_{2}
\end{aligned}
$$

In practice, efficient and cost-effective electrolytic water splitting requires highly active and stable electrocatalysts that can minimize the required energy input (overpotential) [20]. Current state-of-the-art water-splitting technologies utilize noble metal-based electrocatalysts, including platinum (Pt) [21] and ruthenium dioxide $\left(\mathrm{RuO}_{2}\right)$ /iridium dioxide $\left(\mathrm{IrO}_{2}\right)$, which show remarkable activities towards HER and OER, respectively, at low overpotentials [22]. However, the large-scale commercial use of noble-based electrocatalysts has been restricted due to their prohibitive high cost, scarcity, and longterm instability [21, 23]. Therefore, significant research efforts have been directed towards developing robust, highly active, cost-effective, and stable electrocatalysts based on earthabundant elements for water splitting (OER and HER).
Perovskite oxides, a class of transition metal oxides, have emerged as promising electrocatalysts for alkaline OER due to their unique structural, electronic, ionic, and electrocatalytic characteristics [24-26]. The general chemical formula for perovskite oxides is $\mathrm{ABO}_{3}$, where $\mathrm{A}$ is typically occupied by cation with 12-fold coordination and $\mathrm{B}$ is occupied by a cation with 6-fold coordination with oxygen anions [27-32]. Other perovskite oxides with more complex structures include layered perovskites $\left(\mathrm{A}_{n+1} \mathrm{~B}_{n} \mathrm{O}_{3 n+1}\right)$, A-site ordered double perovskite oxides $\left(\mathrm{AA}^{\prime} \mathrm{B}_{2} \mathrm{O}_{6}\right)$, and $\mathrm{B}$-site ordered double perovskite oxides $\left(\mathrm{A}_{2} \mathrm{BB}^{\prime} \mathrm{O}_{6}\right)$ [33]. Compared with other electrocatalytic materials, perovskite oxides are inexpensive because they are based on earth-abundant elements, easy to synthesize, and environmentally benign [27, 33, 34]. Moreover, depending on the type of cations occupying the A and B lattice sites, a plethora of possible perovskite oxides with various stoichiometries can be generated with modulable crystal structures, electrocatalytic activities, electronic, and ionic properties [35]. Yet, despite their promise, perovskite oxides still require further enhancements to outperform noble-based metal catalysts due to their relatively low electronic conductivity.

Several experimental and theoretical studies demonstrated that the catalytic activity of perovskite oxides can be intrinsically modulated through the inductive alteration of oxygen nonstoichiometry to generate oxygen-deficient structures for a myriad of applications [35-39]. Table 1 summarizes various electrocatalysts made of oxygen-defected perovskite oxides that were reported in the literature for OER. Oxygen defects can bring about substantial electrocatalytic performance improvements by (1) tuning the electronic structure and charge transfer, (2) promoting the exsolution of secondary active surface phases, and/or (3) providing moderate adsorption/ desorption behavior of reaction intermediates during water splitting. When the A and B positions are replaced, perovskite oxides maintain their structural stability through undergoing crystal lattice deformations caused by A-site and B-site ionic radii mismatch along a specific direction to construct crystallographic structures (e.g., tetragonal, orthogonal, or trigonal). Thus, oxygen vacancies can be generated on perovskite oxides to a certain extent without rendering a collapsed structure [33].

Reportedly, oxygen vacancies promote the $\mathrm{e}_{\mathrm{g}}$-filling status of the B-cation to alter the electron configuration of the metal cation with a high-spin-state electron occupying the $\mathrm{e}_{\mathrm{g}}$ orbital [40]. Compared with the $\pi$-bonding $\mathrm{t}_{2 \mathrm{~g}}$ orbital, the $\sigma$-bonding $\mathrm{e}_{\mathrm{g}}$ orbital strongly overlaps with the oxygen-containing adsorbates, which facilitates charge transfer between the transition metal cation and the reaction adsorbates $(\mathrm{M}-\mathrm{OH}, \mathrm{M}-\mathrm{O}, \mathrm{M}-\mathrm{OOH}$, $\mathrm{H}_{2} \mathrm{O}$ ), hence accelerating the water splitting kinetics [62]. Generally, optimal OER performance could be obtained by perovskite oxides with an $\mathrm{e}_{\mathrm{g}}$ occupancy close to unity $(\sim 1.00)$ [1]. Besides $e_{g}$ filling, oxygen defects can upshift the oxygen p-band center relative to the Fermi level to promote hybridization and 


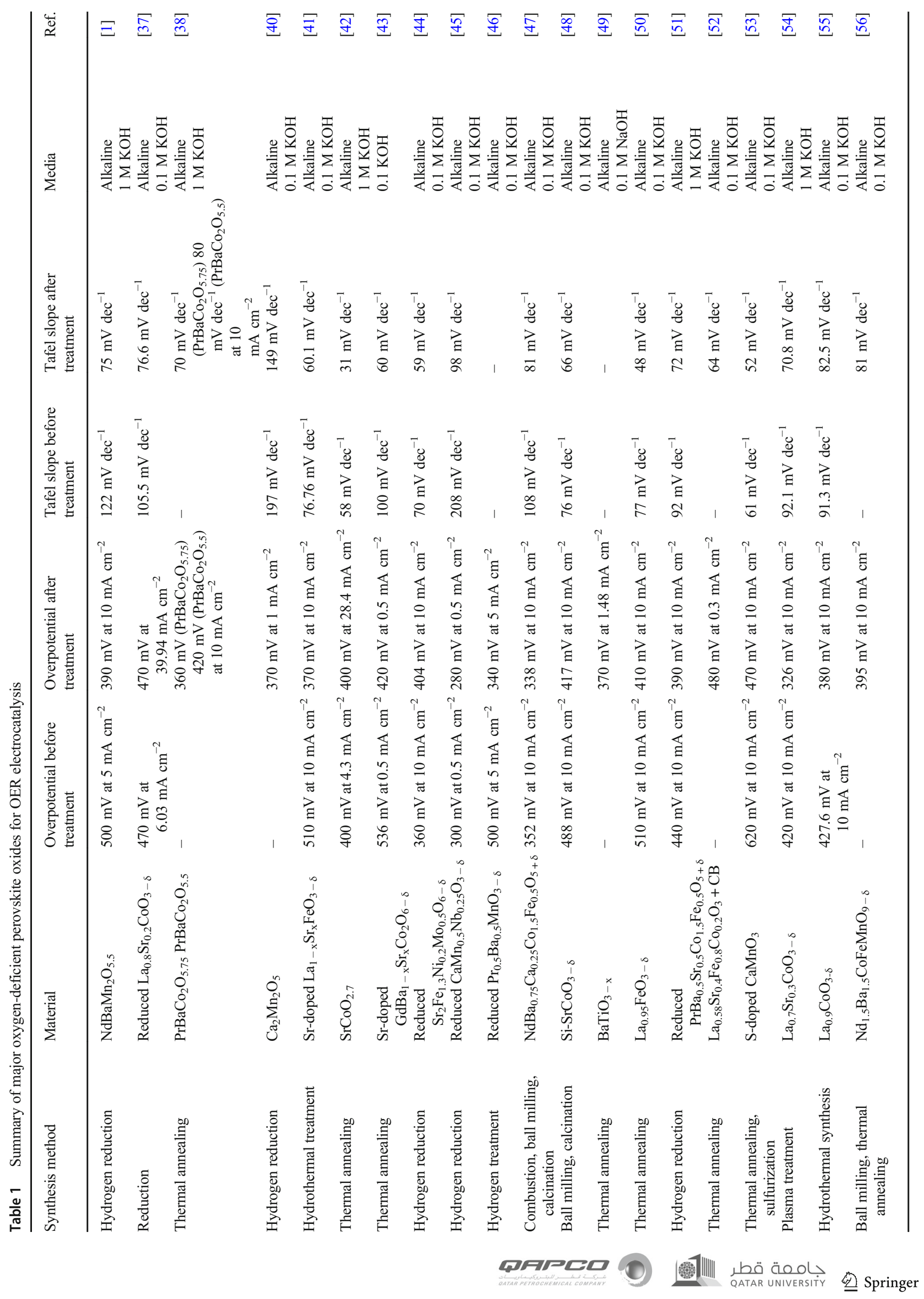




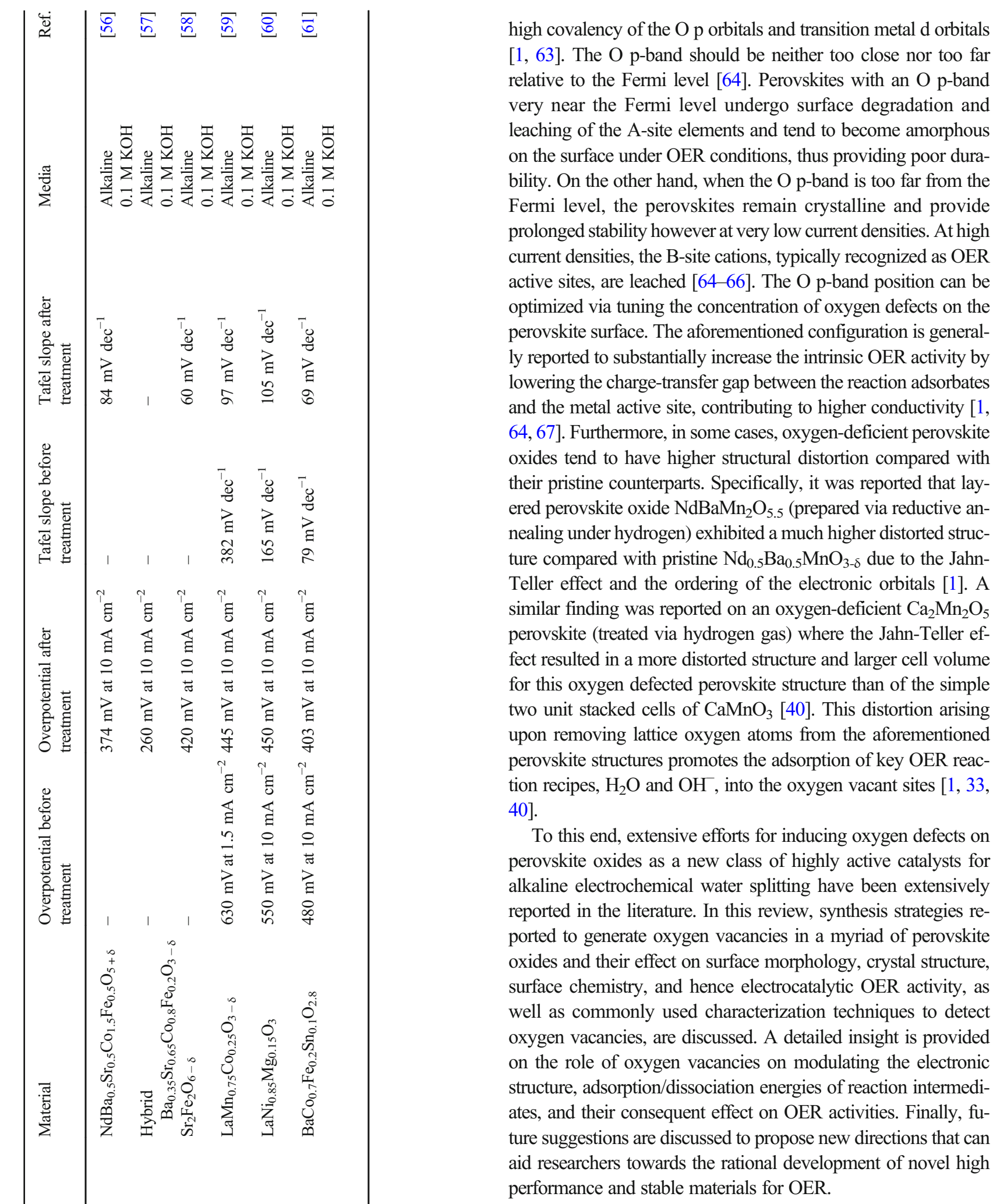

\section{Synthesis methods}

As previously discussed, oxygen defects can effectively enhance the electrocatalytic OER activity of perovskite oxides 
through modulating the surface morphology, electronic structure, and thus their electrocatalytic properties. Various methods have been reported in the literature to preferentially tune the degree of oxygen site defects in the perovskite lattice via in situ or post-synthesis treatments. This section will provide a brief summary of synthesis approaches that have been commonly adopted to induce oxygen vacancies in perovskite oxides. These methods mainly involve gaseous hydrogen reduction, thermal annealing, and elemental doping, whilst very little work has also been reported using other methods, such a plasma treatment, solid-state reduction, and wet-chemical reduction. The degree of which the synthesis method and the process parameters affect the surface morphology, crystallinity, and electrocatalytic OER performance was highlighted.

\subsection{Reduction}

Many studies report the incorporation of oxygen vacancies in perovskite oxides by the facile but very effective hydrogen reduction method due to the high reproducibility of the hydrogen gas [68, 69]. This method can generate oxygen defects either at mild or elevated temperatures [70]. Typically, under a suitable reduction atmosphere, namely $\mathrm{H}_{2}$, due to the high reducibility of the $\mathrm{H}_{2}$ gas, the B-site metal cations of the perovskite oxide are exsolved to form metallic nanoparticles distributed on the surface of the as-prepared pristine perovskite oxides. Consequently, the reduced perovskite oxide will exhibit an enhanced electronic conductivity due to the high conductivity of the metallic particles, which in turn enhances the OER kinetics [44]. Moreover, since oxygen defects can be generated at relatively mild/low-temperature conditions, this avoids sintering or coalescence of the perovskite oxide during the treatment process. This also provides easier control of the reaction kinetics during the synthesis process [40]. However, under excessive reduction of annealing conditions, aggregation of the perovskite oxide nanostructures could occur, leading to a collapsed structure and degraded OER activity [51].

In a representative work by Kim et al., a single crystalphase oxygen-defected $\mathrm{Ca}_{2} \mathrm{Mn}_{2} \mathrm{O}_{5}$ perovskite was synthesized using a low-temperature reduction approach. $\mathrm{Ca}_{2} \mathrm{Mn}_{2} \mathrm{O}_{5}$ was prepared through annealing pristine $\mathrm{CaMnO}_{3}$ (prepared via a sol-gel process) under $5 \% \mathrm{H}_{2} / \mathrm{Ar}$

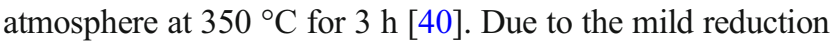
conditions used, no sintering or agglomeration was observed post-annealing. Moreover, $\mathrm{Mn}^{4+}$ ions in $\mathrm{CaMnO}_{3}$ were reduced to $\mathrm{Mn}^{3+}$ which could easily bond with $\mathrm{OH}^{-}$. Reduction annealing has also resulted in crystal structural transformations, where a single crystal phase orthorhombic $\mathrm{Ca}_{2} \mathrm{Mn}_{2} \mathrm{O}_{5}$ coordinated with square pyramid subunits between oxygen and manganese was formed, compared with $\mathrm{CaMnO}_{3}$, which had six-coordinated octahedral subunits connected by six oxygen atoms at the corner sites (Fig. 1a). The unit cell structure of the oxygen-deficient $\mathrm{Ca}_{2} \mathrm{Mn}_{2} \mathrm{O}_{5}$ resulted in the formulation of a molecular level porosity at its internal structure on the oxygen-vacancy sites beyond its apparent structural porosity, which facilitated the adsorption of $\mathrm{OH}^{-}$. The aforementioned modifications ultimately contributed towards enhancing the OER kinetics. OER activity measurements indicated that the onset potential in an alkaline $0.1 \mathrm{M} \mathrm{KOH}$ solution for defective $\mathrm{Ca}_{2} \mathrm{Mn}_{2} \mathrm{O}_{5}$ perovskite was $1.5 \mathrm{~V}$ (vs RHE), compared with its pristine counterpart $\mathrm{CaMnO}_{3}(1.6 \mathrm{~V})$.

Moreover, an oxygen-deficient $\mathrm{Sr}_{2} \mathrm{Fe}_{1.3} \mathrm{Ni}_{0.2} \mathrm{Mo}_{0.5} \mathrm{O}_{6-\delta}$ double perovskite was reported by $\mathrm{Zhu}$ et al. with enhanced OER activity, delivering a current density of $10 \mathrm{~mA} \mathrm{~cm}{ }^{-2}$ at $360 \mathrm{mV}$ compared with its pristine counterpart $(404 \mathrm{mV})$ in an alkaline $0.1 \mathrm{M} \mathrm{KOH}$ solution [44]. The defected perovskite was synthesized via annealing pristine $\mathrm{Sr}_{2} \mathrm{Fe}_{1.3} \mathrm{Ni}_{0.2} \mathrm{Mo}_{0.5} \mathrm{O}_{6}$ ${ }_{-\delta}$ under different atmospheres $\left(\mathrm{Ar}, 5 \% \mathrm{H}_{2} / \mathrm{Ar}\right.$ and $\left.\mathrm{H}_{2}\right)$ at $700{ }^{\circ} \mathrm{C}$ for $3 \mathrm{~h}$. The optimum reduction conditions, showing the highest OER activity, were under $5 \% \mathrm{H}_{2} / \mathrm{Ar}$ as shown in Fig. $2 \mathrm{~b}$. During the annealing process, the metal cations were reduced resulting in mixed-valence states $\left(\mathrm{Mo}^{+5}, \mathrm{Mo}^{+6}, \mathrm{Ni}^{+3}\right.$, $\mathrm{Ni}^{+2}, \mathrm{Fe}^{+2}, \mathrm{Fe}^{+3}$, and $\left.\mathrm{Fe}^{+4}\right)$. Moreover, the presence of metallic $\mathrm{Fe}^{0}$ and $\mathrm{Ni}^{0}$ was detected which improved the electronic conductivity of the catalyst due to the high conductivity of the metallic particles. Post-reduction, the as-synthesized pristine $\mathrm{Sr}_{2} \mathrm{Fe}_{1.3} \mathrm{Ni}_{0.2} \mathrm{Mo}_{0.5} \mathrm{O}_{6-\delta}$ perovskite tetrahedral lattice structure expanded as a consequence of the partial reduction of B-site cations. Furthermore, due to the high reducibility of the hydrogen gas, phase decomposition of the parent pristine perovskite occurred resulting in the exsolution of new Fe-Ni alloy and $\mathrm{Sr}_{4} \mathrm{FeMoO}_{8}$ phases on the reduced $\mathrm{Sr}_{2} \mathrm{Fe}_{1.3} \mathrm{Ni}_{0.2} \mathrm{Mo}_{0.5} \mathrm{O}_{6-\delta}$. The surface area of the defected perovskite increased from 2.6 to $4.0 \mathrm{~m}^{2} \mathrm{~g}^{-1}$ through formulating a porous surface with more catalytic active sites as presented in Fig. 2c and d. As reported, the enhanced OER activity enhancement in reduced $\mathrm{Sr}_{2} \mathrm{Fe}_{1.3} \mathrm{Ni}_{0.2} \mathrm{Mo}_{0.5} \mathrm{O}_{6-\delta}$ is directly related to the aforementioned modifications related to the formation of new active phases and more exposed active sites.

Besides gaseous hydrogen reduction, oxygen defects were also created via solid-state reduction of $\left(\mathrm{Sr}_{1-\mathrm{x}} \mathrm{Ba}_{\mathrm{x}}\right) \mathrm{FeO}_{2}$ perovskite using solid reagents that can thermally release hydrogen $\left(\mathrm{CaH}_{2}, \mathrm{NaH}\right)$ and wet-chemical reduction with $\mathrm{H}_{2} \mathrm{O}_{2}$ aqueous solution on $\mathrm{La}_{0.8} \mathrm{Sr}_{0.2} \mathrm{CoO}_{3-\delta}$ (LSC) [37].

\subsection{Doping}

The substitution of A and/or B site atoms using low valency dopants (e.g., $\mathrm{La}, \mathrm{Sr}, \mathrm{Mg}, \mathrm{Ba}, \mathrm{Ca}$ ) is an effective approach to creating oxygen vacancies. Upon removing a cation atom from the perovskite crystal structure, a negative charge is created resulting in an unbalanced atmosphere. To maintain charge neutrality, an oxygen vacancy is formed by releasing an oxygen atom from the perovskite lattice [33]. The concentration of oxygen vacancies is highly dependent on the degree 


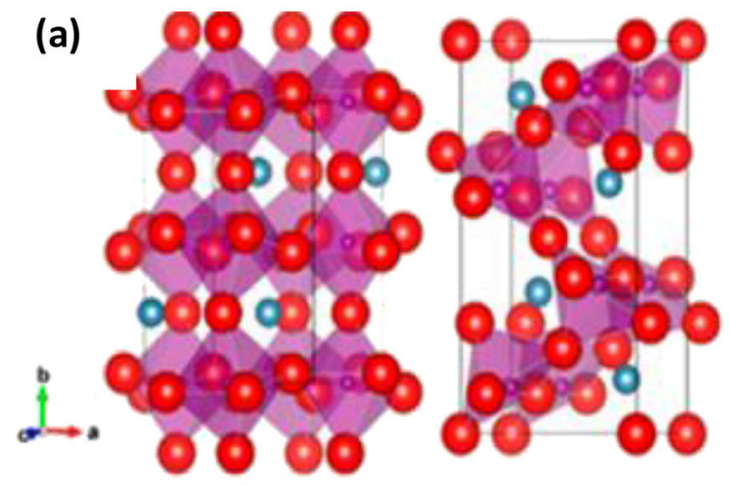

(c)

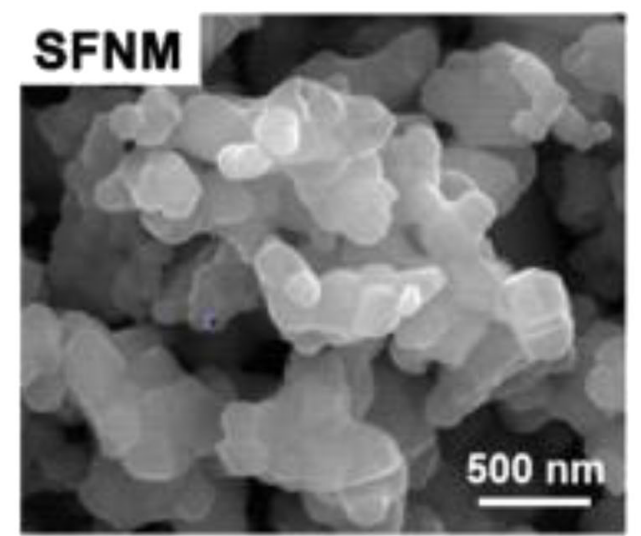

Fig. 1 (a) Unit cell structures of $\mathrm{CaMnO}_{3}$ (left) and $\mathrm{Ca}_{2} \mathrm{Mn}_{2} \mathrm{O}_{5}$ (right). Reproduced from Ref. [40] with permission from the American Chemical Society, Copyright 2014. (b) Performance of SFNM samples treated

of substitution and the type of elemental dopant used, which directly affects the formation energy of the oxygen vacancies. Typically, low formation energy results in a higher degree of oxygen defects. $\mathrm{Sr}$ and $\mathrm{Ca}$ are the most favorable and commonly used alkaline-earth metals to create oxygen vacancies in $\mathrm{ABO}_{3}$-type perovskite oxides $[3,70]$.

She et al. investigated the effect of dopant content on the OER electrocatalytic performance through synthesizing a series of strontium ( $\mathrm{Sr}$ )-doped La-based perovskite oxide catalysts with different compositions of $\mathrm{La}_{1-\mathrm{x}} \mathrm{Sr}_{\mathrm{x}} \mathrm{FeO}_{3-\delta}(\mathrm{x}=0$, $0.2,0.5,0.8$, and 1.0). Sr-doped samples were prepared by LSF-x solid precursors $\left(\mathrm{La}\left(\mathrm{NO}_{3}\right)_{3} \cdot 6 \mathrm{H}_{2} \mathrm{O}, \mathrm{Sr}\left(\mathrm{NO}_{3}\right)_{2}\right.$ $\mathrm{Fe}\left(\mathrm{NO}_{3}\right)_{3} \cdot 9 \mathrm{H} 2 \mathrm{O}$ ) in a solution at $800{ }^{\circ} \mathrm{C}$ for $5 \mathrm{~h}$ (hydrothermal synthesis) [41]. The concentration of oxygen vacancies on $\mathrm{La}_{1-\mathrm{x}} \mathrm{Sr}_{\mathrm{x}} \mathrm{FeO}_{3-\delta}$ increased as the concertation of the of $\mathrm{Sr}^{+2}$ dopant increased, where the highest $\left(\mathrm{O}_{2}{ }^{2-} / \mathrm{O}^{-}\right)$ratio was at $\mathrm{x}=$ 0.8 . The high $\mathrm{O}_{2}{ }^{2-} / \mathrm{O}^{-}$ratio indicates the formation of highly oxidative oxygen species and is a direct measure of surface oxygen vacancies. Moreover, $\mathrm{Fe}$ with different oxidation states $\left(\mathrm{Fe}^{4+}, \mathrm{Fe}^{3+}, \mathrm{Fe}^{2+}\right)$ was detected on the surface and increased with increasing $\mathrm{Sr}^{+2}$ content. Structural analysis revealed that the substitution of $\mathrm{La}^{3+}$ by $\mathrm{Sr}^{2+}$ promoted the lattice structure to change from an orthorhombic structure to a (b)

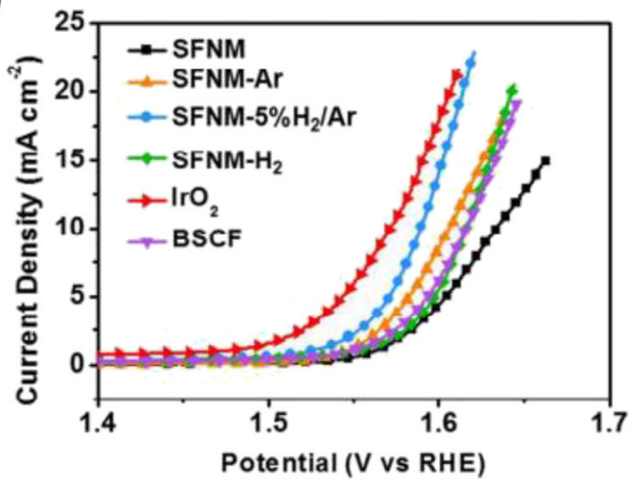

(d)

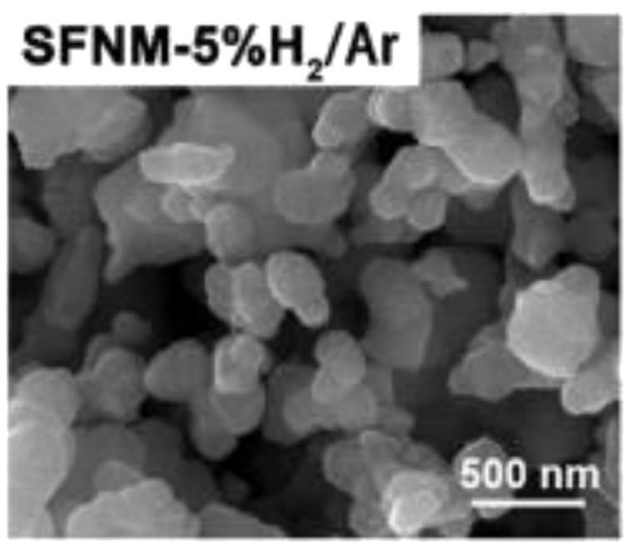

under different atmospheres. SEM images of (c) SFNM and (d) SFNM$5 \% \mathrm{H}_{2} / \mathrm{Ar}$ at $700{ }^{\circ} \mathrm{C}$. Reproduced from Ref. [44] with permission from the Royal Society of Chemistry, Copyright 2017

cubic structure. However, in the case where the $\mathrm{Sr}^{+2}$ dopant fraction was 1.0, a tetragonal lattice was adopted instead of a cubic lattice. Amongst the five samples, $\mathrm{La}_{0.2} \mathrm{Sr}_{0.8} \mathrm{FeO}_{3-\delta}$ (LSF-0.8) exhibited the highest activity towards OER in a $0.1 \mathrm{M} \mathrm{KOH}$ alkaline media, where the overpotential at $10 \mathrm{~mA} \mathrm{~cm}{ }^{-2}$ current density was $370 \mathrm{mV}$ compared with undoped $\mathrm{La} \mathrm{FeO}_{3-\delta}(510 \mathrm{mV}$ ) (Fig. 2a). The cubic structure and the presence of abundant oxygen vacancies both contributed towards enhancing the OER performance [41].

Kim et al. investigated the potential for improving the OER electrocatalytic activity of $\mathrm{NdBa}_{0.75} \mathrm{Ca}_{0.25} \mathrm{Co}_{2} \mathrm{O}_{5+\mathrm{d}}(\mathrm{NBCC})$ double perovskite oxide by doping multiple transition-metal oxides $\left(\mathrm{Fe}^{+2}, \mathrm{Ni}^{+2}, \mathrm{Cu}^{+2}\right.$, and $\left.\mathrm{Mn}^{+2}\right)$ into the B-sites of the perovskite to generate oxygen vacancies. The catalysts were synthesized through combusting solid precursors followed by milling with zirconia balls in ethanol for $24 \mathrm{~h}$, and then calcination for $4 \mathrm{~h}$ at $600{ }^{\circ} \mathrm{C}$, and recalcination for additional $4 \mathrm{~h}$ at $900{ }^{\circ} \mathrm{C}$ [47]. Amongst the prepared catalysts, $\mathrm{NdBa}_{0.75} \mathrm{Ca}_{0.25} \mathrm{Co}_{1.5} \mathrm{Fe}_{0.5} \mathrm{O}_{5+\mathrm{d}}(\mathrm{NBCCFe})$ showed the highest electrocatalytic OER activity with onset potential of $1.568 \mathrm{~V}$ at $10 \mathrm{~mA} \mathrm{~cm}{ }^{-2}$ current density relative to 1.582 , $1.583,1.591$, and $1.599 \mathrm{~V}$ for $\mathrm{NdBa}_{0.75} \mathrm{Ca}_{0.25} \mathrm{Co}_{2} \mathrm{O}_{5+\mathrm{d}}$, $\mathrm{Mn}$ doped $\mathrm{NdBa}_{0.75} \mathrm{Ca}_{0.25} \mathrm{Co}_{2} \mathrm{O}_{5+\mathrm{d}}(\mathrm{NBCCMn}), \mathrm{Ni}$-doped 
Fig. 2 (a) Relationship between specific activities and surface oxygen vacancies and surface $\mathrm{Fe}$ oxidation states for the LSF-x ( $\mathrm{x}=0,0.2,0.5,0.8$, and 1$)$ samples. Reproduced from Ref. [41] with permission from the American Chemical Society, Copyright 2018. Surface morphologies of the catalysts by FESEM: (b) NBCC, (c) NBCCFe, (d) NBCCMn, (e) NBCCNi, and (f) $\mathrm{NBCCCu}$. Reproduced from Ref. [47] with permission from the Royal Society of Chemistry, Copyright 2018
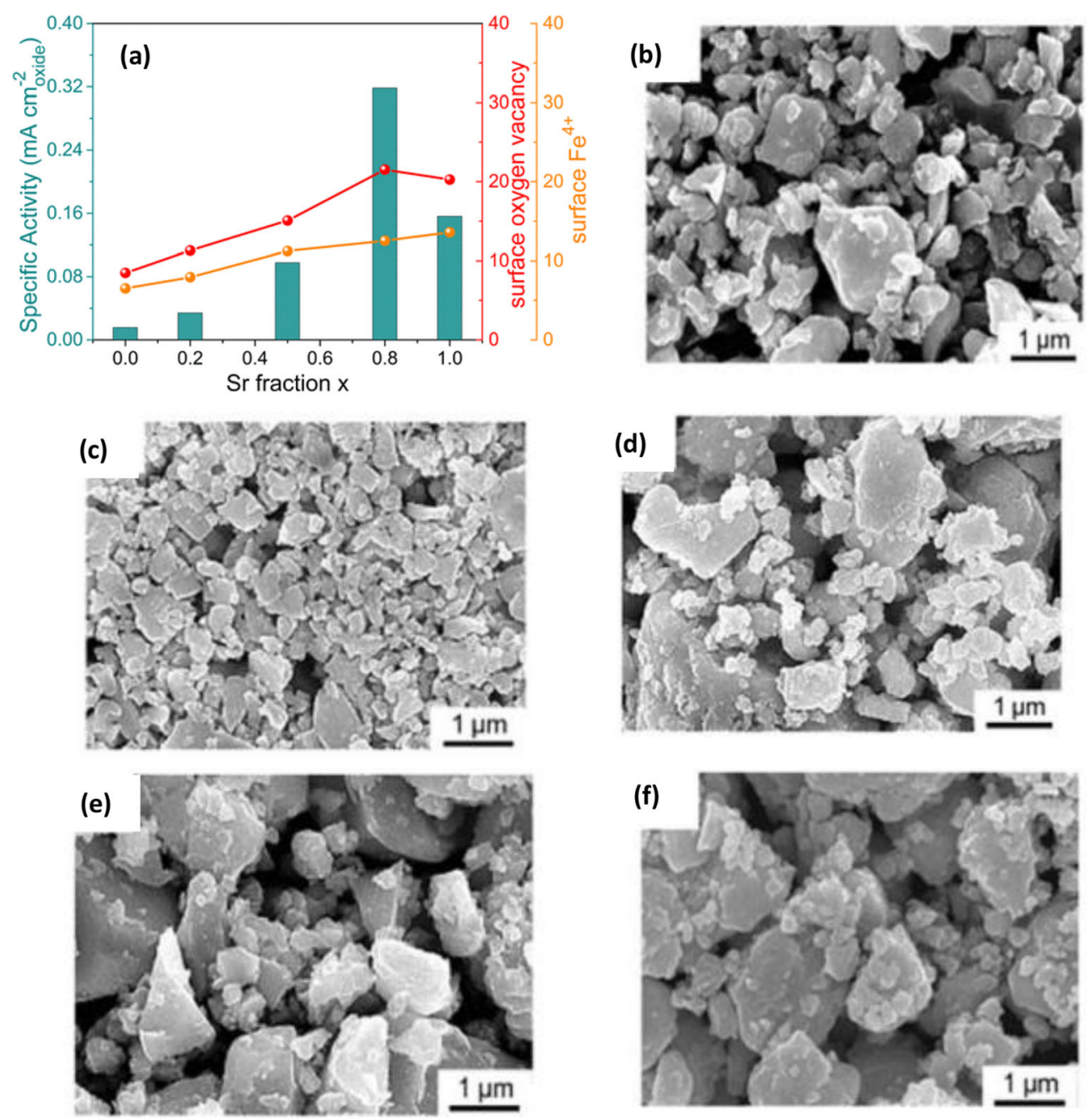

$\mathrm{NdBa}_{0.75} \mathrm{Ca}_{0.25} \mathrm{Co}_{2} \mathrm{O}_{5+\mathrm{d}}$ (NBCCNi), and $\mathrm{Cu}$-doped $\mathrm{NdBa}_{0.75} \mathrm{Ca}_{0.25} \mathrm{Co}_{2} \mathrm{O}_{5+\mathrm{d}}(\mathrm{NBCCCu})$, respectively. Doped NBCCFE, NBCCMn, and NBCCNi were crystallized with an orthorhombic structure that was very similar to NBCC. On the other hand, $\mathrm{Cu}$ doping resulted in the transformation of the orthorhombic $\mathrm{NdBa}_{0.75} \mathrm{Ca}_{0.25} \mathrm{Co}_{2} \mathrm{O}_{5+\mathrm{d}}$ structure to a tetrahedral structure due to the large difference between the ionic radii and the electronic structure of $\mathrm{Cu}$ and $\mathrm{Co}$. Based on morphological analysis, the agglomerated $\mathrm{NdBa}_{0.75} \mathrm{Ca}_{0.25} \mathrm{Co}_{2} \mathrm{O}_{5+\mathrm{d}}$ morphology was precluded upon doping as shown in the SEM images in Fig. 2b-f. Moreover, the metallic substitutions resulted in the generation of porous structures where NBCCFe exhibited the highest exposed surface area $\left(2.8 \mathrm{~m}^{2} \mathrm{~g}^{-1}\right)$ compared with NBCC $\left(1.9 \mathrm{~m}^{2} \mathrm{~g}^{-1}\right)$, $\mathrm{NBCCCu}\left(1.8 \mathrm{~m}^{2} \mathrm{~g}^{-1}\right)$, NBCCNi $\left(1.9 \mathrm{~m}^{2} \mathrm{~g}^{-1}\right)$, and $\operatorname{NBCCMn}\left(2.2 \mathrm{~m}^{2} \mathrm{~g}^{-1}\right)$.

In contrast to thermal annealing/calcination, doping via hydrothermal synthesis requires low processing temperatures (normally below $300{ }^{\circ} \mathrm{C}$ ). This allows for precise control of the particle size, shape, and crystallinity of perovskite oxide via tuning the synthesis process conditions. Moreover, due to the mild temperature conditions used via this synthesis route, agglomerated free powders can be produced. Thus, electrocatalysts with high specific area and unique favorable morphologies towards OER can be generated $[3,71]$.

\subsection{Thermal treatment}

Calcination/thermal annealing under low oxygen partial pressures or vacuum at elevated temperatures is a fairly simple and direct approach to produce non-stoichiometric perovskite oxides [72]. However, catalysts produced using this method can contain agglomerated large particles of various sizes and morphologies, as well as phase impurities [3]. Moreover, the high calcination temperatures associated with this synthesis route can result in sintering of the perovskite oxide catalyst which may result in a poor microstructure, reduction in surface area and coarsening of the catalyst powders, hence low electrochemical OER performance [73]. Therefore, the catalyst powder post-calcination is typically refined or ball-milled to separate the particle aggregate and obtain a catalyst powder with enhanced particle size distribution [52].

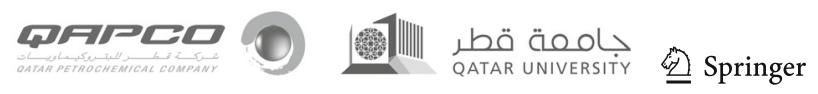


Miao et al. studied the role of oxygen vacancy concentration in enhancing the electrocatalytic OER activity of $\mathrm{PrBaCo}_{2} \mathrm{O}_{6-\delta}$ perovskite. Two oxygen-deficient perovskites with different oxygen vacancy concentrations $(\delta)$ of 0.25 $\left(\mathrm{PrBaCo}_{2} \mathrm{O}_{5.75}\right)$ and $0.5\left(\mathrm{PrBaCo}_{2} \mathrm{O}_{5.5}\right)$ were synthesized. The catalysts were produced by sintering $\mathrm{PrBaCo}_{2} \mathrm{O}_{6-\delta}$ powders (produced via a sol-gel method) in air at $1000{ }^{\circ} \mathrm{C}$ for $10 \mathrm{~h}$ followed by annealing in pure $\mathrm{N}_{2}$ at 250 and $600{ }^{\circ} \mathrm{C}$ for 30 min to obtain $\mathrm{PrBaCo}_{2} \mathrm{O}_{5.75}$ and $\mathrm{PrBaCo}_{2} \mathrm{O}_{5.5}$. Interestingly, higher oxygen vacancies $(\delta=0.5)$ have detrimentally affected the intrinsic OER activity of the cobalt oxide-based perovskite. $\mathrm{PrBaCo}_{2} \mathrm{O}_{5.75}$ depicted faster OER kinetics in $1 \mathrm{M} \mathrm{KOH}$ alkaline media with a low overpotential of $360 \mathrm{mV}$ to achieve a current density of $10 \mathrm{~mA} \mathrm{~cm}$ as compared with $\mathrm{PrBaCo}_{2} \mathrm{O}_{5.5}(420 \mathrm{mV})$. As the oxygen vacancy introduced, both $\mathrm{PrBaCo}_{2} \mathrm{O}_{5.75}$ and $\mathrm{PrBaCo}_{2} \mathrm{O}_{5.5}$ displayed similar particle morphologies as shown in Fig. 3a and b. However, at $(\delta=0.5)$, as the oxygen atoms were dislodged from the octahedral $\mathrm{Co}^{4+} \mathrm{O}_{6}$ in $\mathrm{PrO}_{1-\delta}$ layers, a pyramidal $\mathrm{Co}^{3+} \mathrm{O}_{5}$ was formed, resulting in an alternative arrangement of pyramidal $\mathrm{Co}^{3+} \mathrm{O}_{5}$ and octahedral $\mathrm{Co}^{3+} \mathrm{O}_{6}$ within the $\mathrm{PrO}_{1}$ ${ }_{-\delta}$ layers (Fig. 3c). Consequently, this ordered structure reduced the cobalt oxidation states and caused a spin-state transition from high-spin to low-spin states for the cobalt ions, which both significantly increase charge resistance hence hampering the OER kinetics [38].

In another work by Chen et al., an oxygen-deficient $\mathrm{BaTiO}_{3-\mathrm{x}}$ perovskite denoted as (BTO-1300VAC) was synthesized using a sol-gel method followed by vacuum thermal treatment at $1300{ }^{\circ} \mathrm{C}$ for $2 \mathrm{~h} \mathrm{[49].} \mathrm{For} \mathrm{comparison} \mathrm{purposes,}$ BTO-950AIR was produced via calcinating pristine $\mathrm{BaTiO}_{3}$ in air at $950{ }^{\circ} \mathrm{C}$ for $2 \mathrm{~h}$. Moreover, benchmark $\mathrm{IrO}_{2}$ nanoparticles (denoted as Ir-550) were synthesized via a sol-gel method followed by calcination in oxygen at $550{ }^{\circ} \mathrm{C}$ for $2 \mathrm{~h}$ to directly compare its OER activity with the as-synthesized oxygen-deficient perovskite. The measured surface areas of BTO-1300VAC and $\mathrm{IrO}_{2}$ were 24.7 and $21.1 \mathrm{~m}^{2} \mathrm{~g}^{-1}$, respectively. Post-annealing, small agglomerations of the $\mathrm{BaTiO}_{3}-\mathrm{x}$ particles were observed due to the high-temperature conditions used in the calcination process (Fig. 3d). On the other hand, BTO-950AIR formulated highly agglomerated particles due to the bonding reaction in air at $950{ }^{\circ} \mathrm{C}$ (Fig. 3e). Moreover, in contrast to BTO-950AIR and $\mathrm{BaTiO}_{3}$ which exhibited pure tetrahedral phases $\left(\mathrm{t}-\mathrm{BaTiO}_{3}\right)$, mixture of tetragonal and hexagonal $\mathrm{BaTiO}_{3}\left(\mathrm{~h}-\mathrm{BaTiO}_{3}\right)$ phases was exsolved upon annealing. Results of electrochemical OER measurements in $0.1 \mathrm{NaOH}$ electrolyte solution showed that the BTO-1300VAC sample generates larger current density than $\mathrm{Ir}-550$ at relatively low potential $(<1.6 \mathrm{~V})$. However, the measured current density for Ir-550 at high potentials ( $>$ $1.6 \mathrm{~V}$ ) rapidly increases and exceeds that for BTO-1300VAC. This was attributed to the higher conductivity and active site density of $\mathrm{IrO}_{2}$ over perovskite oxides. BTO-950AIR showed a very low electrocatalytic OER activity with an onset of around $1.8 \mathrm{~V}$.

\section{Characterization techniques for oxygen vacancies}

As will be discussed in detail in Section 4, the presence of oxygen vacancies results in notable changes in the performance of a perovskite electrocatalyst. Such enhancements in electrocatalytic activities emerge from both structural and electronic modulations to the material. For instance, the omission of several surface and sub-surface oxygen vacancies can lead to structural instabilities and phase changes - as will be discussed later. Crystallographic techniques, such as XRD, can indirectly probe the presence of oxygen vacancies in structurally complex perovskites upon slight phase expansions or compressions that result in a more stable configuration. Furthermore, it has been found that amongst the main changes that perovskites undergo upon the induction of oxygen vacancies, modulation in electronic and magnetic properties as a result of metallic-oxygen species near the surface and charge neutrality effects, respectively, are witnessed. This section will briefly overview some of the most prominent and advanced characterization techniques employed in literature to effectively identify the presence of oxygen-deficient perovskites. It is worth noting that the techniques discussed have been utilized for the characterization of oxygen vacancies in a myriad of transition metal oxides (TMOs); however, this section will solely review relevant endeavors in identifying oxygen-deficient perovskites.

The ordering of oxygen vacancies has been investigated in TMOs due to the observed influence that vacancy ordering has on magnetic and transport properties. Advanced microscopy using high resolution transmission electron microscopy (HRTEM) and scanning transmission electron microscopy (STEM) can be utilized to directly probe evident surface structural distortions as a result of surface lattice realignment upon the induction of oxygen vacancies. Klie et al. reported the use of high-angle annular dark-field (HAADF) STEM technique to convey patterned ordering of oxygen-deficient $\mathrm{La}_{0.5} \mathrm{Sr}_{0.5} \mathrm{CoO}_{3-\delta}$ (LSCO) on $\mathrm{SrTiO}_{3}$ (STO) and $\mathrm{LaAlO}_{3}$ (LAO) substrates in the [74] orientation [75]. Oxygen vacancies were indirectly found to be ordered in LCO thin films grown on a (001) oriented STO substrate, whereby the contrast is resulting from oxygen vacancy ordering [76]. This is presented in Fig. 4a. Furthermore, Fig. 4b shows enlarged LaLa distances as a result of the induced oxygen vacancy planes.

The induction of oxygen vacancies in pristine $\mathrm{Pr}_{0.5} \mathrm{Ba}_{0.5} \mathrm{MnO}_{3-\delta}(\mathrm{PBM})$ by direct annealing in $\mathrm{H}_{2}$ was reported by Ciucci et al., and clear changes to the crystalline phase of the reduced H-PBM are conveyed through XRD comparison between both samples [46]. Phase transition from 
Fig. 3 SEM images of (a) $\mathrm{PrBaCO}_{2} \mathrm{O}_{5.75}$ and (b) $\mathrm{PrBaCo}_{2} \mathrm{O}_{5.5}$. (c) Schematic crystal structures of $\mathrm{PrBaCo}_{2} \mathrm{O}_{5.75}$ (left) and $\mathrm{PrBaCo}_{2} \mathrm{O}_{5.5}$. (right) Color codes: $\mathrm{Pr}$, orange; $\mathrm{Ba}$, green; Co, blue; $\mathrm{O}$, red. Reproduced from Ref. [38] with permission from the Royal Chemical Society, Copyright 2019. SEM images for (d) BTO1300VAC $\left(1300^{\circ} \mathrm{C}\right.$ in vacuum $)$ and (e) BTO-950AIR $\left(950^{\circ} \mathrm{C}\right.$ in air) samples. Reproduced from Ref. [49] with permission from Elsevier, Copyright 2015
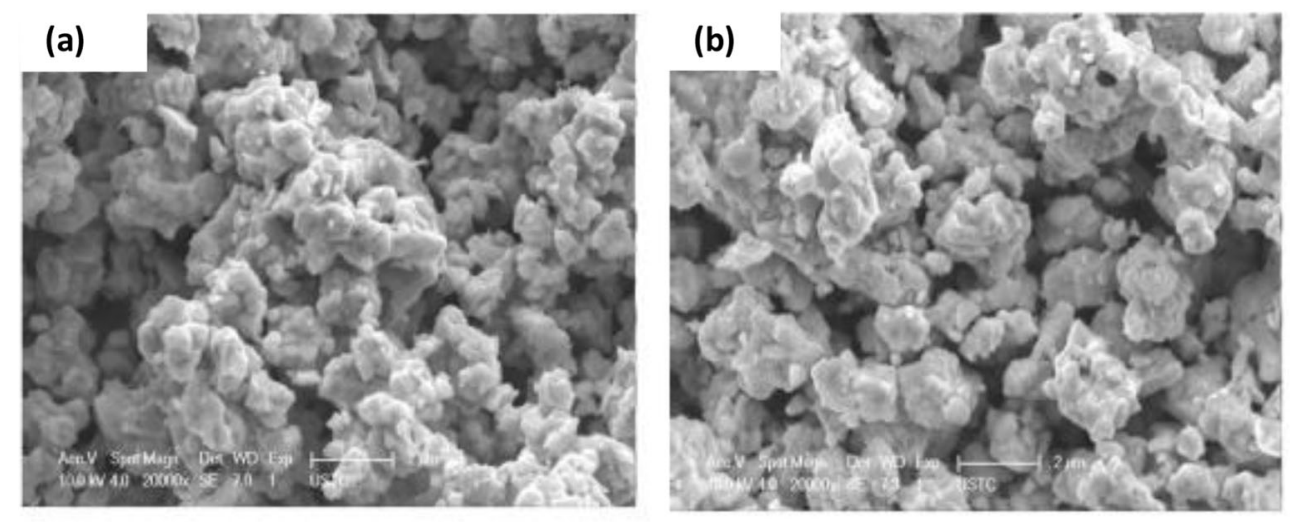

(c)
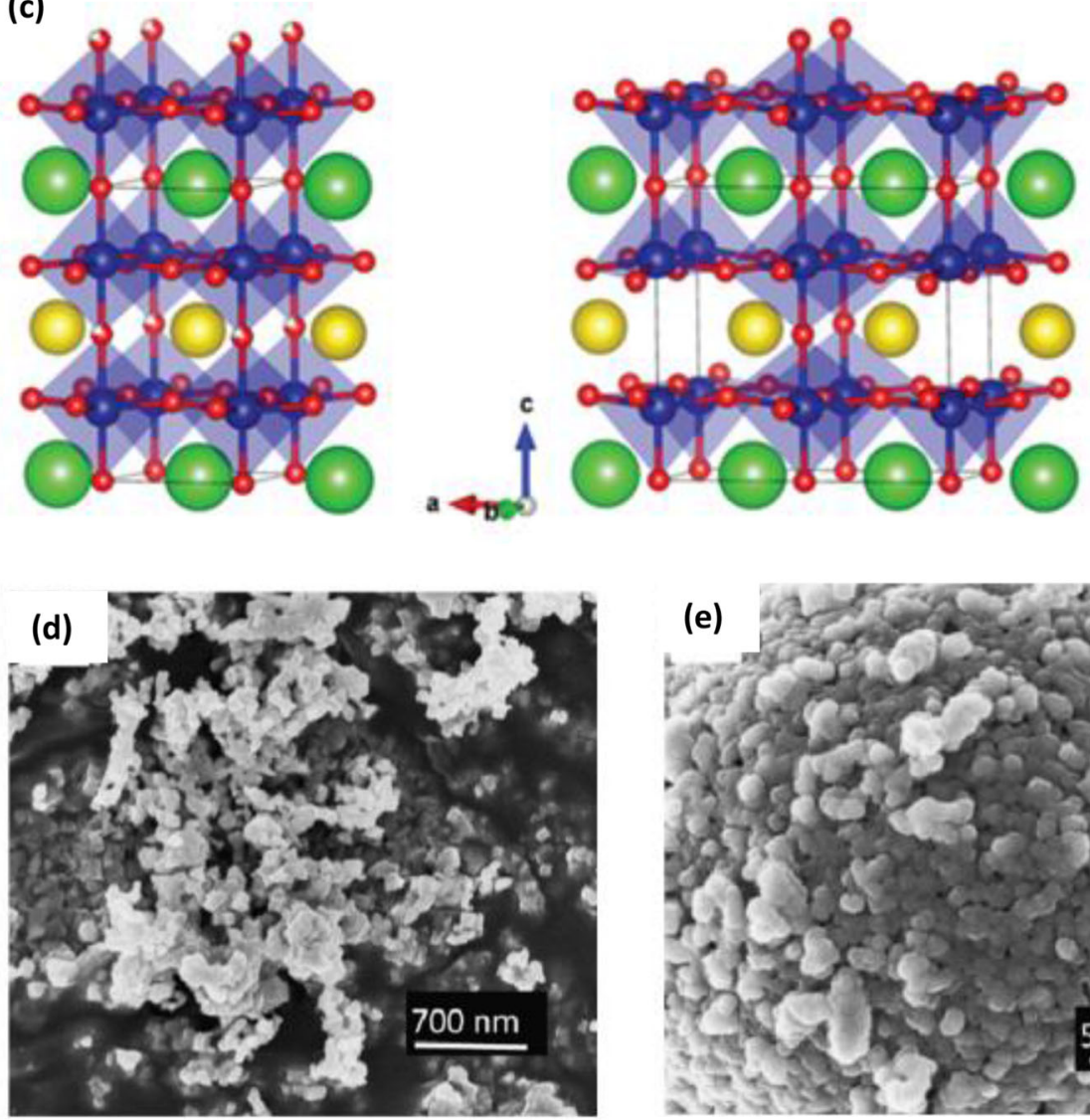

the cubic structure with randomly distributed $\mathrm{Pr} / \mathrm{Ba}$ sites to the ordered one can be depicted from Fig. $4 \mathrm{c}$ and $\mathrm{d}$, respectively. Reduction of the pristine perovskite can also result in a lowering of transition metal ions to a lower valence states and the decomposition of the original coupled material into its constituent oxide and metals. However, the latter of this can be ruled out in the case of H-PBM based on XRD patterns not showing phases of the standalone components of the perovskite. XRD technique is one of a few characterization methods that was reported in several studies to determine the presence oxygen vacancies in perovskites $[77,78]$. Upon reduction-and consequent introduction of oxygen vacancies - peak shifts to lower angles infer an increase in out-of-plane lattice parameter (c) and cell volume. This is attributed to atomic underbonding orbitals located in nonbinding orbitals of transition metals due to the presence of large number of electrons upon oxygen vacancy formation [70].

Neutron powder diffraction (NPD) is another effective approach for depicting oxygen vacancies in perovskites. The neutrons utilized in NPD technique have higher penetration depth than the penetration depth of X-rays, which allows for bulk characterization and a higher sensitivity towards oxygen atoms. This proves effective for studying oxygen-deficient perovskites [79-82]. For instance, Chen et al. employed the

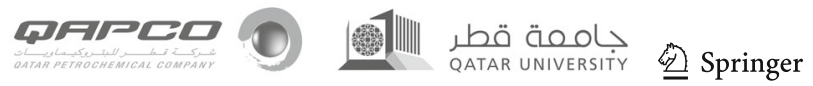



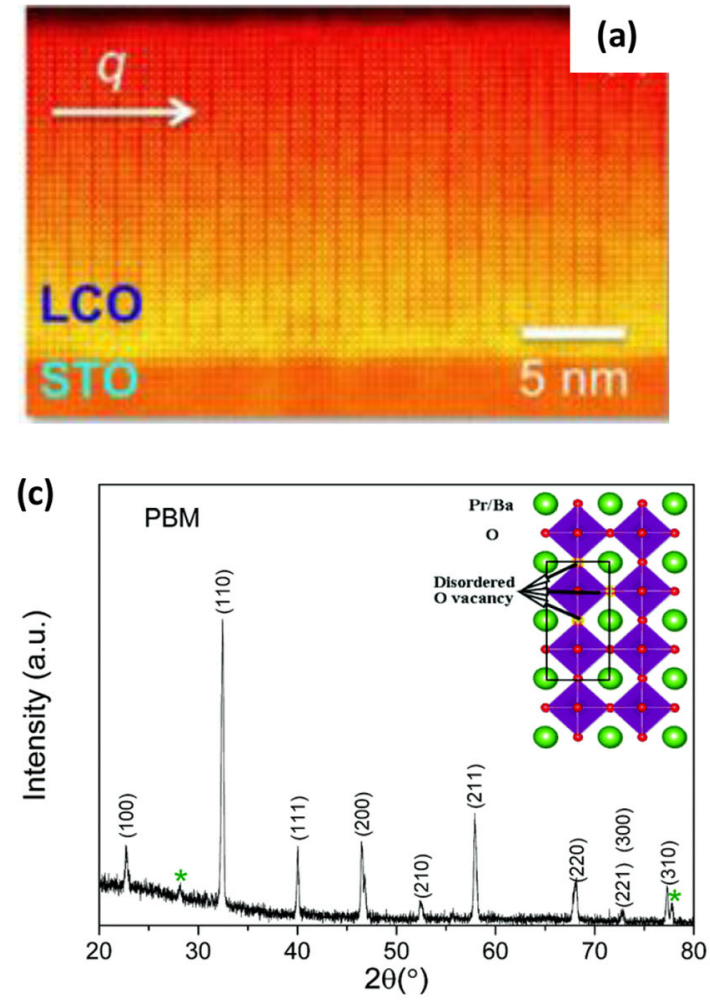

Fig. 4 High-resolution, high-angle annular dark field (HAADF) image of a LCO thin film $15 \mathrm{~nm}$ thick, grown on a (001) oriented STO substrate, showing the contrast resulting from the $\mathrm{O}$-vacancy ordering. The direction of the modulation vector, q, is marked with an arrow. (a) Map of the in-plane distance between first La neighbors $(\Delta x)$ in $(\mathbf{b})$, along the

Rietveld method in NPD to exemplify potential active sites in oxygen-deficient $\mathrm{BaTiO}_{3}$ [49]. It was also used to study the oxygen atom site occupancies in structural models as free variables in the refined spectra. It was thus determined that tetragonal phases did not promote vacancy formation; however, the oxygen site occupancies in hexagonal $\mathrm{BaTiO}_{3}$ phase refined to 0.92(3)-representing $\mathrm{BaTiO}_{2.76}$. Such detailed phase analysis is essential for oxygen vacancy engineering, since as will be discussed later, not all phases have the same enthalpic favorability towards vacancy formation and represent different binding energies towards reaction intermediates.

$\mathrm{X}$-ray photoelectron spectroscopy (XPS) is perhaps the most employed characterization method for indirectly determining the presence of oxygen vacancies in the surface of oxide-based materials. XPS works by determining the surface electronic and chemical states of a material through effectively measuring the contrast in outermost layer electrons and their atomic cores' binding energies. A reduced material with oxygen vacancies tends to always have surface oxygen vacancies since reduction initially attacks surface lattice oxygen, and consequently through the deconvolution of the $\mathrm{O} 1 \mathrm{~s}$ spectra of a reduced material, the determination of vacant sites can be inferred. Due to charge neutrality restrictions, neighboring
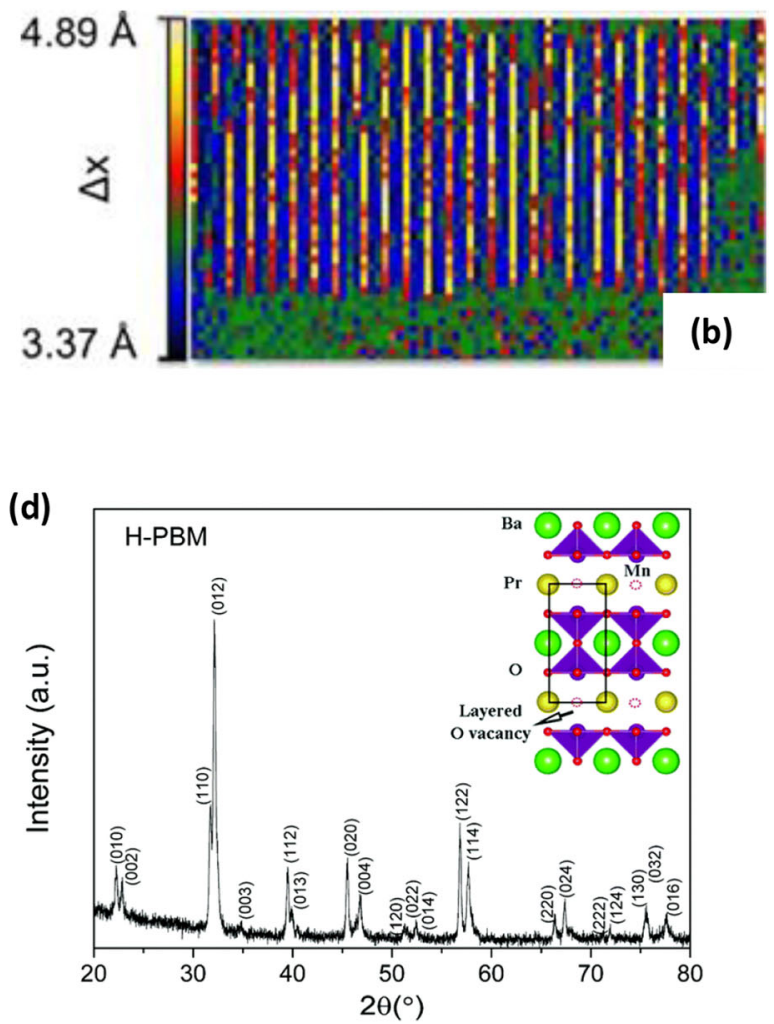

direction parallel to q. O-deficient planes are characterized by enlarged La-La distances. Reproduced from Ref. [76] with permission from the American Physical Society, Copyright 2014 . PXRD patterns of (c) assynthesized PMB and (d) H-PBM. Reproduced from Ref. [46] with permission from The Royal Chemical Society, Copyright 2016

cationic metallic sites to the oxygen vacancy site lower their valence oxidation states. Yang et al. fabricated $\mathrm{Sr}_{2} \mathrm{Fe}_{1.3} \mathrm{Ni}_{0.2} \mathrm{Mo}_{0.5} \mathrm{O}_{6-\delta}$ (SFNM) with oxygen vacancies through calcination in argon (Ar), $5 \% \mathrm{H}_{2} / \mathrm{Ar}$, and $\mathrm{H}_{2}$ atmospheres [44]. The resultant samples were denoted SFNM-Ar, SNFM-5\%, and SNFM- $\mathrm{H}_{2}$, respectively. XPS results presented in Fig. 5 show the different degrees of oxygen vacancies the materials possess, which was directly correlated to the order in OER performance. The deconvoluted peaks of the Ni 2p, Fe 2p, and Mo 3d spectra in the modified SFNM samples of Fig. 5a-c show a clear lowering of oxidation states upon reduction in $\mathrm{H}_{2}$ and $5 \% \mathrm{H}_{2} / \mathrm{Ar}$ atmospheres. This is in order to cope with surface charge electroneutrality conditions for more thermodynamic surface-stable reduced structure. Furthermore, the reduction of $\mathrm{B}$-site ions $(\mathrm{Ni}, \mathrm{Fe}$, and $\mathrm{Mo}$ ) to a lower oxidation state leads to a decrease in the binding energy of the lattice oxygen, whilst the oxygen vacancies greatly enhance the binding energy of the lattice oxygen. Figure $5 \mathrm{~d}$ shows two main deconvoluted peaks for the $\mathrm{O} 1 \mathrm{~s}$ spectra of different SFNM samples. Essentially, a peak at $529 \mathrm{eV}$ corresponds to lattice oxygens, and the second peak at a binding energy of $531 \mathrm{eV}$ corresponds to adsorbed oxygen species (i.e., $\mathrm{O}_{2}^{-}, \mathrm{O}^{-}$), which is an indirect identifier of oxygen 
Fig. 5 XPS spectra of SFNM, SFNM-Ar, SFNM-5\% H2/Ar, and SFNM-H2: (a) Ni 2p; (b) Fe 2p; (c) Mo 3d; and (d) O 1s. Reproduced from Ref. [44] with permission from The Royal Chemical Society, Copyright 2017 (a)

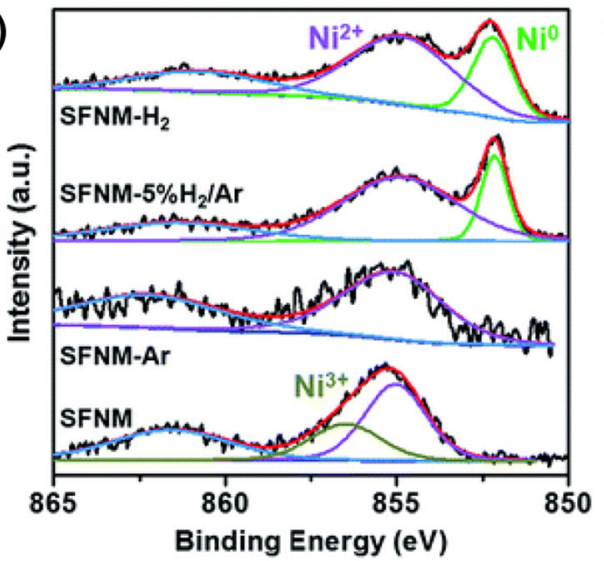

(c)

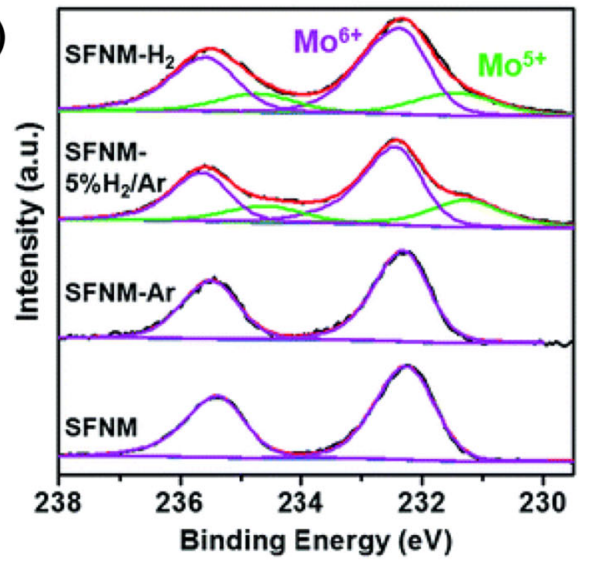

(b)

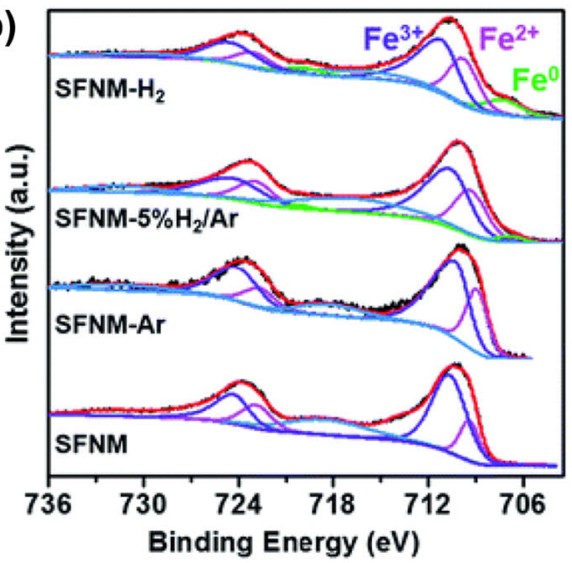

(d)

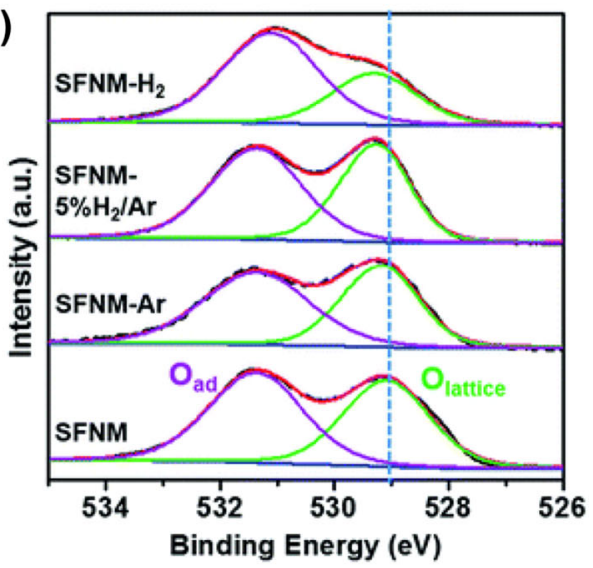

vacant sites. The adsorbed oxygen electrophilic species could attack regions of $\mathrm{H}_{2} \mathrm{O}$ molecules with the highest electron densities - in effect enhancing OER kinetics.

The presence of oxygen vacancies in a perovskite leads to the trapping of unpaired electrons, which can be detected through electron paramagnetic resonance (EPR). EPR as a characterization technique can shed light on both bulk and surface unpaired electrons. However, a disadvantage in using EPR as a sole method to determine the presence of oxygen vacancies lies in the fact that it innately is unable to differentiate between oxygen vacancies and other types of defects that can lead to the same unpairing effect of electrons. Eichel performed a thorough investigation of several transition metaldoped perovskites through the analysis of high-frequency EPR [83]. The presence of oxygen deficiencies in all the studies materials was confirmed by g-parameters close to 2.00 . For instance, Mn-doped $\mathrm{Bi}_{0.5} \mathrm{Na}_{0.5} \mathrm{TiO}_{3} \mathrm{EPR}$ analysis found a gparameter of 2.0015. Numerous findings of g-parameters approaching 2.00 for oxygen-deficient perovskites and transition metal oxides (TMOs) co-confirmed with other oxygen vacancy characterization techniques have been reported in recent years [84-86]. Acquiring information pertaining to molecular vibrations and rotations via Raman spectroscopy is another tool continued to be adopted for identifying oxygendeficient materials and perovskites in general [87-90].
Changes in lattice molecular vibrational nodes due to novel bond configurations give rise to Raman shifts. Oxygen vacancies can thus result in observed Raman shifts or the creation of new peaks. Moreover, anionic vacancies tend to modify band structures - as can be seen from DFT band structure calculations of oxygen-deficient materials. Such band structure changes can lead to potential alternate paths for excited electron generation and recombination-which occurs from lightdirected electron excitation during photoluminescence (PL) readings [85, 91]. Peak position and intensity of the PL spectra show easily identifiable changes upon such electronic modulations from oxygen vacancies [92].

The aforementioned analyses show a varying degree of accuracy and reliability in identifying oxygen vacancies in perovskites, and oxygen-deficient TMOs generally. Nevertheless, based on the most prominently utilized and accepted techniques, XPS and STM are generally used to detect surface chemistry changes and physical imaging of oxygen vacancies, respectively. Although the creation of surface oxygen vacancies is more important than bulk vacancies in photocatalytic and electrocatalytic applications, some applications may find bulk vacancies important. For the latter applications, both XPS and STM may not be very useful since they characteristically capture the first few layers of a material's surface. It is generally a common practice to perform at least two 
characterization techniques for an accepted claim of oxygen vacancies. Moreover, phase characterization techniques, such as XRD, are only useful for identifying oxygen deficiencies when the target material is crystalline in nature. Therefore, factoring for the fact that every technique exhibits some form of limitation, it is considered best practice to use multiple characterization techniques on the same material to undoubtedly prove the presence of oxygen vacancies.

\section{Advantageous intrinsic effects of oxygen vacancies in perovskites}

Induction of oxygen vacancies in perovskites has been reported to advantageously enhance the physicochemical properties directly related to lower overpotentials for electrochemical OER through water splitting. Literature shows a clear sparked increase in interest towards oxygen-deficient perovskitesnot only due to an apparent improvement in performance. Oxygen vacancies in perovskites are amongst the easiest anionic or Schottky defects to be induced and reasonably controlled due to their low formation energies. Other types of defects, such as interstitial or Frenkel defects, tend to be more challenging to control. Furthermore, due to a growing interest in oxygen-deficient metal oxides, both experimental and computational studies have been devoted to mechanistically investigate how oxygen vacancies alleviate an initially high OER overpotential for a pristine sample. Quintessentially for perovskites, three main areas were found to be modulated upon induction of oxygen vacancies - namely, changes in the relaxed configuration structure, improvements in bulk charge transport, and favorable inflection of the electronic structure.
In their recent work, Tavassol et al. synthesized an oxygendeficient $\mathrm{Sr}_{2-\mathrm{x}} \mathrm{Ca}_{\mathrm{x}} \mathrm{Fe}_{2} \mathrm{O}_{6-\delta}$ perovskite for tunable surface reactivity towards OER [93]. This was undertaken by changing the A-site composition $\left(\mathrm{A}=\mathrm{Sr}_{2}-{ }_{\mathrm{x}} \mathrm{Ca}_{\mathrm{x}}\right)$ and the oxygen vacancy content $(\delta)$. Figure 6 a shows a clear direct proportionality between the degree of oxygen vacancy $(\delta)$ and the reduction in OER overpotential. Similar findings have been reported with other oxygen-deficient perovskites. For instance, Fig. $6 \mathrm{~b}$ also shows a direct correlation between the most oxygen-deficient $\mathrm{La}_{1-} \mathrm{Sr}_{\mathrm{x}} \mathrm{CoO}_{3-\delta}$ and the highest OER activity for the optimum material $\mathrm{SrCo}_{2.7}$ [42].

Researchers have utilized so-called "activity descriptors" for predicting the intrinsic behavior of transition metal oxides (TMOs) and their activity towards OER. Essentially, two main descriptors that are related to oxygen vacancies have been frequently investigated to merit their utilization; they are the $e_{g}$ occupancy and the difference between the metal dband center and the oxygen $p$-band center $\left(\Delta E_{d-p}\right)[94,95]$. Both these descriptors can be thought of as modulators of electronic structure. In their recent and very thorough work, Kim et al. primarily focused on investigating the relationship between oxygen vacancies, $\Delta \mathrm{E}_{\mathrm{d}-\mathrm{p}}$, and apparent overpotential for $\mathrm{Sm}_{0.5} \mathrm{Sr}_{0.5} \mathrm{CoO}_{3-\delta}$ (SSC) [96]. The authors were motivated by the fact that previous studies solely focused on the effect of $\delta$ on catalytic activity without much consideration of the electronic structure of cobalt in SSC. They performed a detailed study using X-ray absorption spectroscopy (XAS) characterization for recording changes to the oxidation state of Co upon different degrees of oxygen vacancies $(\delta)$ were induced, and density functional theory (DFT) calculations to reveal more accurate reaction pathways. Early TMs (i.e., V, Mn, and $\mathrm{Cr}$ ) show higher $\mathrm{M}_{\mathrm{d}}$ compared with the

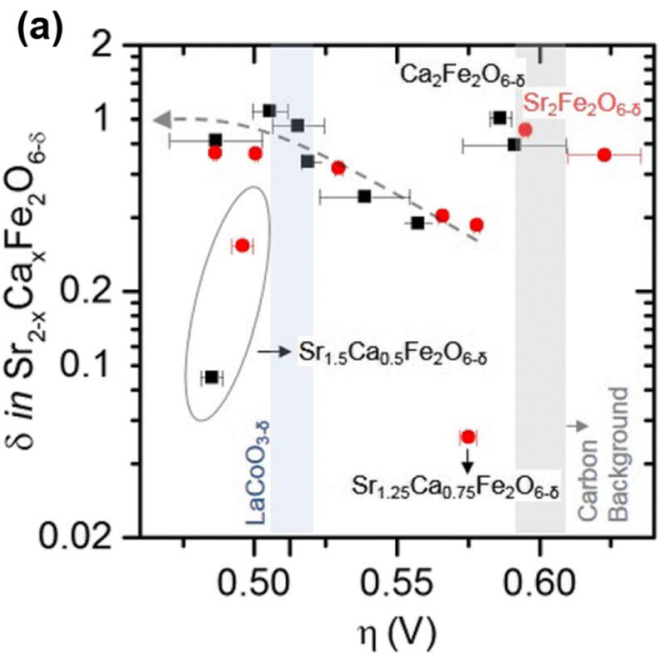

Fig. 6 (a) $\delta$ vs $\eta(V)$ plot for the compounds synthesized in the series of $\mathrm{Sr}_{2-\mathrm{x}} \mathrm{Ca}_{\mathrm{x}} \mathrm{Fe}_{2} \mathrm{O}_{6-\delta}$ under air (red) and argon (black) atmospheres. Gray and light blue bars show activities associated with carbon background and LCO, respectively. $\eta$ is calculated from the thermodynamic potential of OER, $1.23 \mathrm{~V}$ vs RHE, at $2 \mathrm{~mA} \mathrm{~cm}^{-2}$. Dashed line is drawn to guide the eye. Reproduced from Ref. [93] with permission from the American

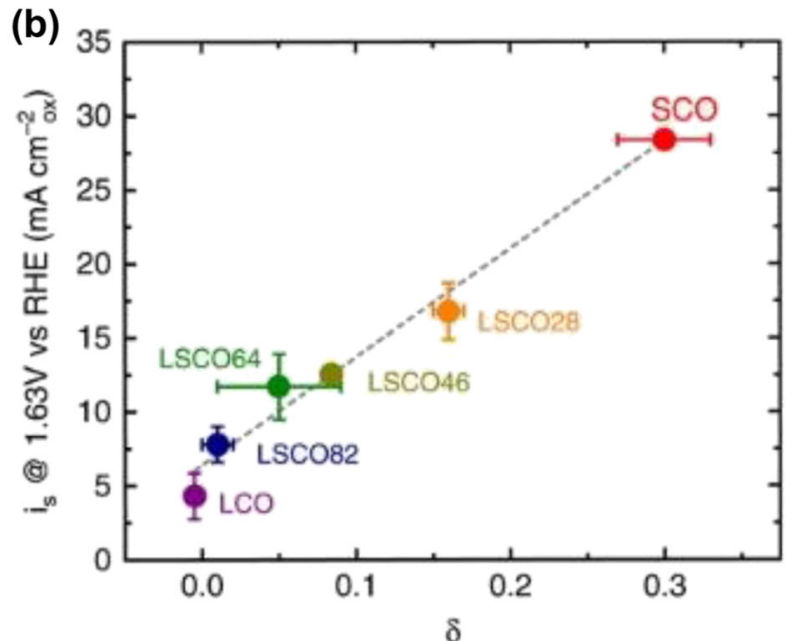

Chemical Society, Copyright 2019. (b) Correlation of oxygen evolution activity for $\mathrm{La}_{1-x} \mathrm{Sr}_{\mathrm{x}} \mathrm{CoO}_{3-\delta}$ with the vacancy parameter $\mathrm{d}$. The vacancy parameter is indicative of the underlying electronic structure where vacancies are generated when there is significant Co $3 d$ and $O 2 p$ band overlap. Reproduced from Ref. [42] with permission from Springer Nature, Copyright 2016 
oxygen $\mathrm{p}$-band center $\left(\mathrm{O}_{\mathrm{p}}\right)$, whilst late transition metals (i.e., $\mathrm{Ni}, \mathrm{Co}$, and $\mathrm{Cu}$ ) show lower $\mathrm{M}_{\mathrm{d}}$ compared with $\mathrm{O}_{\mathrm{p}}$ [97]. Thus, OER performance can be increased by increasing $\mathrm{M}_{d}$ which in effect decreases the $\Delta E_{d-p}$. This relationship is displayed in Fig. 7a. The $\delta$ value was varied between 0 and 0.2 (with 0.1 increments), and the content of oxygen vacancies in the oxygen-deficient SSC samples was determined using iodometric titrations. DFT analyses confirmed that OER activity was improved upon vacancy introduction due to lifting the metal d-band centers $\left(\mathrm{M}_{\mathrm{d}}\right)$ and reducing $\left(\Delta \mathrm{E}_{\mathrm{d}-\mathrm{p}}\right)$.

Figure $7 \mathrm{~b}$ shows that the OER onset potential was found to decrease from 1.62 to $1.54 \mathrm{~V}$ (vs RHE) at $1 \mathrm{~mA} / \mathrm{cm}^{2}$ with an increase in oxygen vacancy content $(\delta)$. Furthermore, a DFT investigation pertaining to the placement of the oxygen vacancies was performed, whereby as can be seen from Fig. 7c, the overpotential is lowest when the anionic vacancy is in the first layer. This is since $\Delta \mathrm{E}_{\mathrm{d}-\mathrm{p}}$ is lowest when oxygen vacancies are induced in the top layer. The reaction coordinate diagram in Fig. 7d shows how the placement of oxygen vacancy in the topmost layer lowers the thermodynamic barrier for each intermediate step - namely, the rate-determining step (RDS). Similar findings of increased OER activity corresponding to a decrease in $\Delta E_{d-p}$ have been reported over the years [98, 99].

(a)

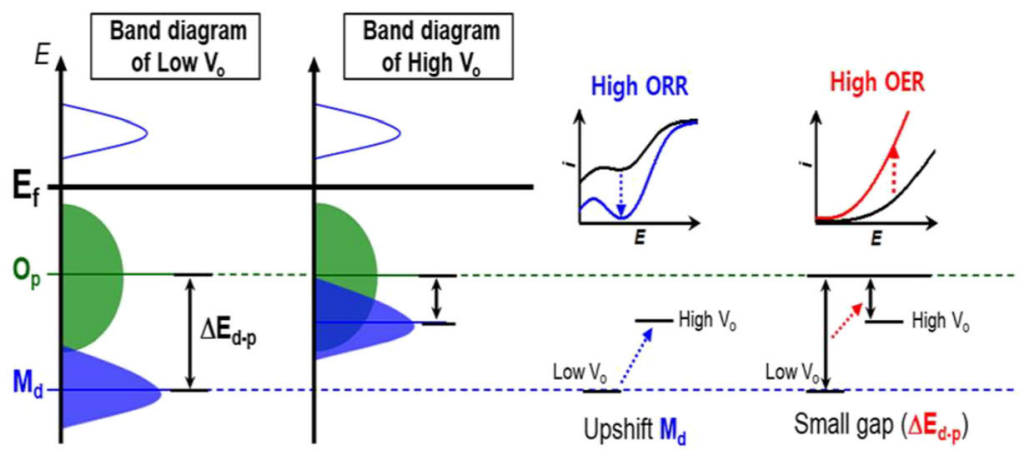

(b) (RRDE) Experiment
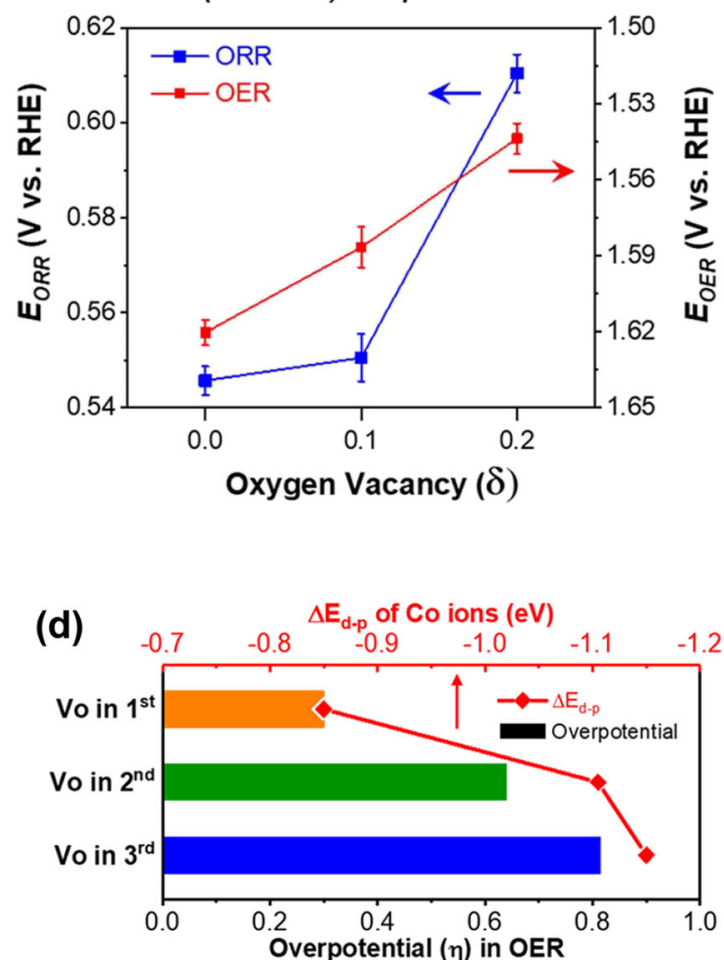

Fig. 7 (a) Schematic rigid band diagrams of the late transition metal oxide. (b) Variation in the Md energy levels and the energy level differences between $\mathrm{Md}$ and $\mathrm{Op}\left(\mathrm{E}_{\mathrm{d}-\mathrm{p}}\right)$ with the number of oxygen defects in the SSC. All band centers are relative to the Fermi level. (c) Overpotential and $E_{d-p}$ values of the OER depending on the oxygen (c)

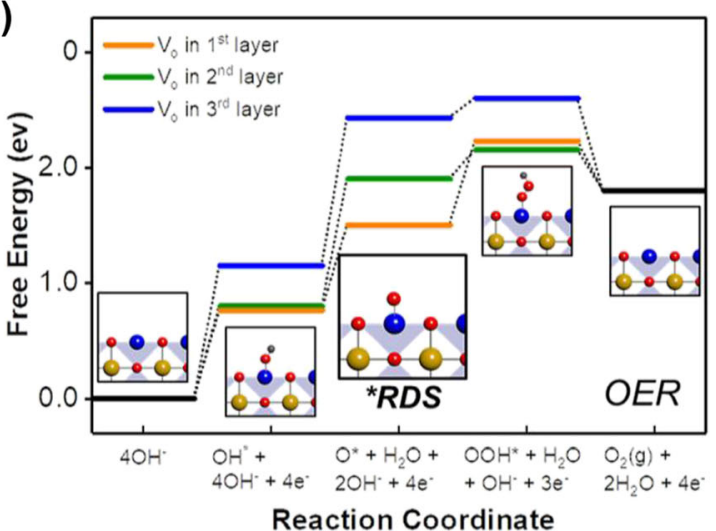

vacancy position for the first, second, and third layers. (d) Calculated free energy profiles of the ORR and OER on the (100) surface of SSC in alkaline media, where the asterisk symbols in reaction coordinates represent the adsorption state. Reproduced from Ref. [96] with permission from the American Chemical Society, Copyrights 2020 
Suntivich et al. described the e eccupancy descriptor to strongly affect the rate-determining step (RDS) in OER [62]. The authors reported that $\mathrm{Ba}_{0.5} \mathrm{Sr}_{0.5} \mathrm{Co}_{0.8} \mathrm{Fe}_{0.2} \mathrm{O}_{3-\delta}$ (BSCF) yielded an order of magnitude more prominent intrinsic activity than benchmark $\mathrm{IrO}_{2}$ for OER in alkaline $0.1 \mathrm{M} \mathrm{KOH}$ media. The formulation of this optimized material was undertaken by systematically examining ten different transition metal oxides (TMOs). A notable finding of an apparent volcano-plot pattern emerged relating the occupancy of the $\mathrm{e}_{\mathrm{g}}$ orbitals (i.e., $\left.\mathrm{dz}^{2}, \mathrm{dx}^{2}-\mathrm{y}^{2}\right)$ in surface TM cations and occupancy of the $3 \mathrm{~d}$ electrons. The peak of the volcano plot shows that an $\mathrm{e}_{\mathrm{g}}$ occupancy close to unity, reflecting high covalency between TM-Oxygen bonds, corresponds to optimum OER activity. This is presented in Fig. 8a, whereby the performance of BSCF is close to the apex of the volcano plot. Suntivich argues that in the case where the $e_{g}$ occupancy is less than unity,

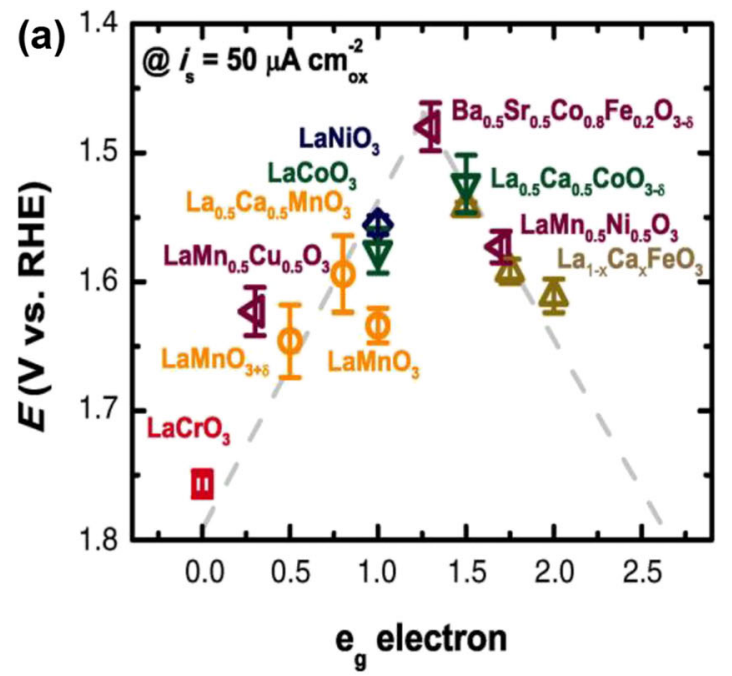

(c)

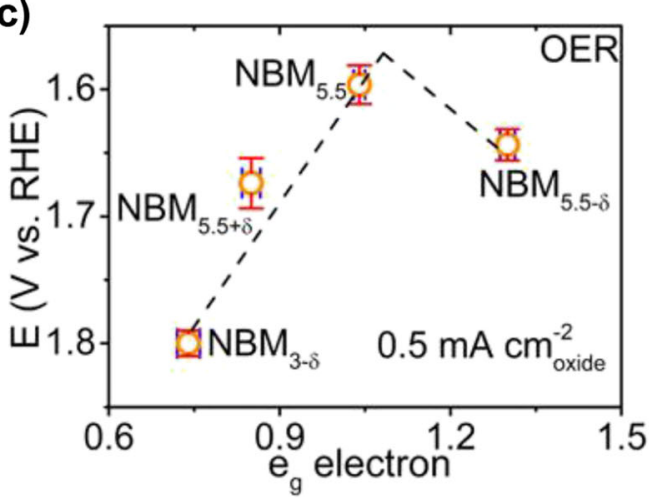

Fig. 8 (a) The relation between the OER catalytic activity, defined by the overpotentials at $50 \mu \mathrm{A} \mathrm{cm}^{-2}$ ox of OER current, and the occupancy of the $\mathrm{e}_{\mathrm{g}}$-symmetry electron of the transition metal ( $\mathrm{B}$ in $\mathrm{ABO}_{3}$ ). Data symbols vary with type of $\mathrm{B}$ ions $(\mathrm{Cr}$, red; $\mathrm{Mn}$, orange; $\mathrm{Fe}$, beige; $\mathrm{Co}$, green; $\mathrm{Ni}$, blue; mixed compounds, purple), where $\mathrm{x}=0,0.25$, and 0.5 for Fe. Error bars represent SDs of at least three independent measurements. The dashed volcano lines are shown for guidance only. Reproduced from Ref. [62] with permission from The American Association for the Advancement of Science, Copyright 2011. (b) Schematic representation the formation of the peroxide ion from preceding oxyhydroxide would be the RDS. In contrary, if the $\mathrm{e}_{\mathrm{g}}$ occupancy is greater than unity, the formation of $\mathrm{O}-\mathrm{O}$ bonds in the OOH* OER intermediate would be the RDS. The relevance of this comes in the finding that introduction of oxygen vacancies in perovskites actually brings the $\mathrm{e}_{\mathrm{g}}$ occupancy close to unity. For instance, $\mathrm{Wu}$ et al. found that oxygen-deficient $\mathrm{CaMnO}_{3}$ perovskite samples showed activity enhancements up to a certain point $-\mathrm{e}_{\mathrm{g}}$ filling approaching 1 [36]. They also related this enhancement to a dual improvement in intrinsic conductivity of the material. The optimum $\mathrm{Yb}$-doped and oxygen-deficient $\mathrm{Yb}_{0.1} \mathrm{Ca}_{0.9} \mathrm{MnO}_{3}$ sample showed 100 times higher activity than the original undoped and un-reduced $\mathrm{CaMnO}_{3}$ sample. This was attributed to optimal $\mathrm{Mn} \mathrm{e}_{\mathrm{g}}$ filling state as high as 0.81 and better conductivity. Similarly, the activity of $\mathrm{Mg}$-doped oxygen-deficient $\mathrm{LaNiO}_{3}$ (LNO)

\section{(b)}
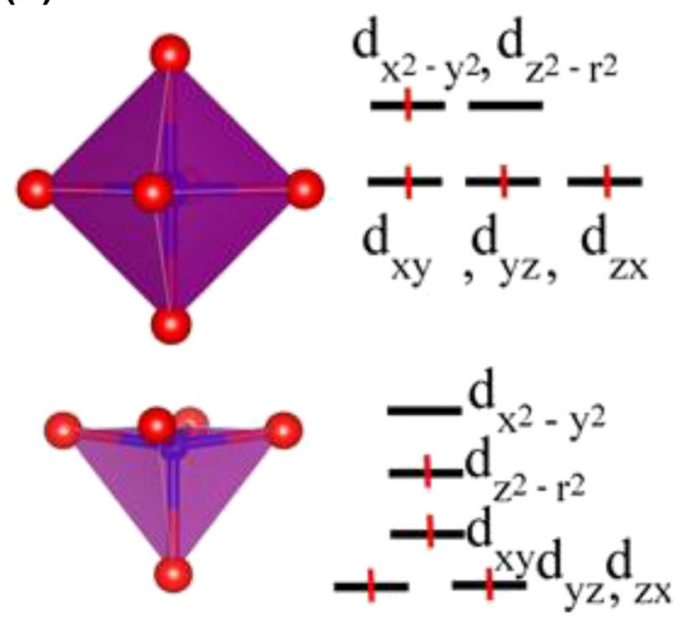

(d)

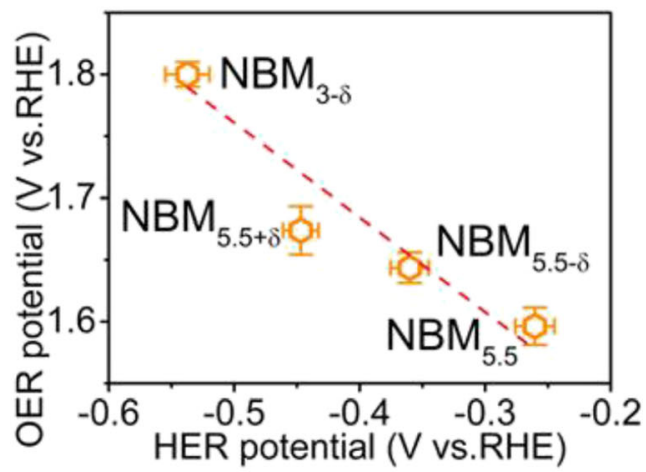

of the octahedral and square pyramidal forms for Mn cations, with different electron distributions in the corresponding crystal-field split dorbit. (c) Dependence of OER activity on the occupancy of the $e_{g}$ orbit. (d) Relationship between OER and HER activity, both of which are defined as the potential to deliver a current density of $0.5 \mathrm{~mA} / \mathrm{cm}^{2}$ of oxide. The error bar is the standard deviation calculated from three separate experiments. The dashed volcano line is displayed only for reference. Reproduced from Ref. [1] with permission from the American Chemical Society, Copyright 2018 
nanofibers fabricated through electrospinning was shown to outperform the pristine LNO counterpart [60]. Typically, $\mathrm{Ni}^{2+}$ presence in LNO leads to an $\mathrm{e}_{\mathrm{g}}$ occupancy greater than unity. However, STXM-XANES analysis of the Mg-doped LNO (LNMO) sample showed an increase in the $\mathrm{Ni}^{3+}$ state, whereby an increased $\mathrm{Ni}^{3+} / \mathrm{Ni}^{2+}$ ratio could lead to an e eccupancy approaching unity and enhancing OER activity. Moreover, Ciucci et al. reported the HER activity of oxygen-deficient $\mathrm{NbBaMn}_{2} \mathrm{O}_{6-\delta}$ (NMB) fabricated via direct hydrogen reduction treatment [1]. The optimum oxygen-deficient $\mathrm{NbBaMn}_{2} \mathrm{O}_{5.5}\left(\mathrm{NMB}_{5.5}\right)$ achieved a better overall water splitting stable activity at large potentials $(>1.75 \mathrm{~V})$ compared with benchmark $\mathrm{IrO}_{2}$ and $\mathrm{RuO}_{2}$ catalysts. Figure $8 \mathrm{~b}-\mathrm{d}$ shows $\mathrm{NMB}_{5.5}$ yielding the most favorable alkaline HER and OER activity compared with other NMB fabricated perovskites, ascribed to near unity $e_{\mathrm{g}}$ occupancy, along with favorable $\Delta \mathrm{E}_{\mathrm{d}-\mathrm{p}}$ levels and structural distortions.

It has also been found that some activity descriptors, such as $e_{g}$ occupancy and $\Delta E_{d-p}$ levels, can be impacted through modulation of lattice strains, especially in perovskites. Although most studies tend to discuss oxygen deficiencies and lattice strains in perovskites independently, Liu et al. found a symbiotic relation between the two [78]. They expressed these effects intrinsically through monitoring $\mathrm{e}_{\mathrm{g}}$ occupancies in oxygen-deficient $\mathrm{La}_{0.7} \mathrm{Sr}_{0.3} \mathrm{CoO}_{3-\delta}$ (LSC) thin films grown atop single crystal substrates $\left(\mathrm{SrTiO}_{3}(\mathrm{STO})\right.$ and $\mathrm{LaAlO}_{3}$ (LAO), with in-plane tensile and compressive strain, respectively). Experimental results and DFT calculations illustrated that higher oxygen deficiencies in LSC/STO samples corresponded to lower OER activities, compared with LSC/ LAO with fewer vacancies and higher compression strains. This is an example of disadvantageous synergy between tensile strains in perovskites facilitating oxygen vacancies, which leads to higher $\mathrm{e}_{\mathrm{g}}$ occupancies and $\Delta \mathrm{E}_{\mathrm{d}-\mathrm{p}}$ levels in the $\mathrm{LSC} /$ STO sample and results in diminished electrochemical activity. On the contrary, Petrie et al. showed that $4.2 \%$ epitaxial/ tensile strains in strontium cobaltite to enhance oxygen deficiencies at benign synthesis conditions and lead to an orderof-magnitude improvement in intrinsic OER activity [100]. Such modest tensile strains allowed unconventionally low anionic concentrations not found in the unstrained bulk cobaltite and lead to an increased $\mathrm{Co}^{3+}$ ratio which admits the $\mathrm{e}_{\mathrm{g}}$ occupancy closer to unity.

Based on the discussion thus far, an ideal OER perovskite should have an $\mathrm{e}_{\mathrm{g}}$ value approaching unity, but also high conductivity [62]. However, most pristine oxides do not have both ideal $\mathrm{e}_{\mathrm{g}}$ occupancies and good conductivities. Wu et al. related the 100 times increase in OER activity of $\mathrm{Yb}_{0.1} \mathrm{Ca}_{0.9} \mathrm{MnO}_{3}$ treated at $350{ }^{\circ} \mathrm{C}$ (CYMO-350), compared with pristine $\mathrm{CMO}$, to both the enhancement of $\mathrm{e}_{\mathrm{g}}$ occupancy and conductivity [36]. This was achieved by sequential doping and hydrogen treatment at elevated temperatures. Figure $9 \mathrm{a}, \mathrm{b}$ represents the OER polarization curves and the identified $e_{g}$ occupancy vs. conductivity of the representative samples investigated. Similar findings have been numerously reported whereby the induction of oxygen vacancies has led to an apparent substantial increase in perovskite's conductivity. For example, Shao et al. reported a silicon-incorporated perovskite as an OER material, which showed a 12.8-fold increase in oxygen diffusivity and two orders of magnitude increase in conductivity - matching the attained 10-fold enhancement in intrinsic OER activity [48]. Previously reported pristine $\mathrm{SrCoO}_{3-\delta}(\mathrm{SCO})$ with relatively high and stable OER performance was modified with silicon. Pristine SCO showed a conductivity of $2 \mathrm{~S} \mathrm{~cm}^{-1}$, whilst a remarkable value of $198 \mathrm{~S} \mathrm{~cm}^{-1}$ was recorded for the silicon modified oxygendeficient sample (Si-SCO). The comparative alkaline $(0.1 \mathrm{M}$ $\mathrm{KOH})$ OER polarization curve in Fig. 9c corresponds to Tafel slope values of 76 and $66 \mathrm{mV} \mathrm{dec}{ }^{-1}$ for pristine $\mathrm{SCO}$ and Si$\mathrm{SCO}$, respectively. This is also a good indication of improved OER kinetics since lower Tafel slopes imply significantly increased currents at only moderate increments of overpotential. Furthermore, Ciucci et al. developed a novel bifunctional perovskite catalyst towards OER and ORR, whereby $\mathrm{Nb}$ was substituted in the $\mathrm{Mn}$ site of $\mathrm{CaMnO}_{3}(\mathrm{CMO})$ to yield $\mathrm{CMNO}$ and inducing oxygen vacancies in the structure upon hydrogen treatment $\left(\mathrm{H}_{2}-\mathrm{CMNO}\right)$ [45]. Hydrogen treatment was found to not only affect the phase structure of CMNO, but it also enhanced the concentration of oxygen vacancies based on XPS analysis. This has contributed to more effective electronic transport through conductivity enhancement between CMO $\left(3.33 \times 10^{-3} \mathrm{~S} \mathrm{~m}^{-1}\right)$ and $\mathrm{H}_{2}$-CMNO $(2.19 \times$ $10^{-2} \mathrm{~S} \mathrm{~m}^{-1}$ ). Figure $9 \mathrm{~d}$ shows the substantial difference in mass activity as a performance parameter between pristine $\mathrm{CMO}, \mathrm{CMNO}$, and the highly oxygen-deficient $\mathrm{H}_{2}-\mathrm{CMNO}$. Other works showing OER performance exceeding that of benchmark $\mathrm{IrO}_{2}$ have been reported over the years $[101,102]$.

Shao et al. systematically investigated structural distortions of $\mathrm{La}_{1-x} \mathrm{Sr}_{\mathrm{x}} \mathrm{FeO}_{3-\delta}$ (LSF) upon varying degrees of Sr-doping in $\mathrm{LaFeO}_{3-\delta}$ (LF) which induced proportional increases in oxygen deficiencies based on XPS analysis [41]. The content of Sr-doping was varied from $\mathrm{x}=0,0.2,0.5,0.8$, and 1.0 , with the alkaline $(0.1 \mathrm{M} \mathrm{KOH})$ OER performance evaluated using an RDE at $1600 \mathrm{rpm}$ increasing in the order LF (LSF-0), SF (LSF-1.0), LSF-0.2, LSF-0.5, and LSF-0.8 corresponding to an increase in Sr-doping and oxygen deficiency character in the doped perovskite. Based on XRD analysis performed, structural distortions and phase configurational changes occur upon progressive doping as can be observed from Fig. 10a. Furthermore, the corresponding electrochemical impedance spectra (Nyquist plot) in Fig. 10b shows highest resistivity in the LF and SF samples having orthorhombic and tetragonal structures, respectively. Similarly, Cheng et al. correlated structural variations with $\mathrm{Sr}$-doping in $\mathrm{La}_{1-} \mathrm{Sr}_{\mathrm{x}} \mathrm{CoO}_{3}$ (LSC) [103]. The authors found a relatively abrupt phase transformation from a rhombohedral $\left(\mathrm{LaCoO}_{3}\right)$ to a cubic structure 

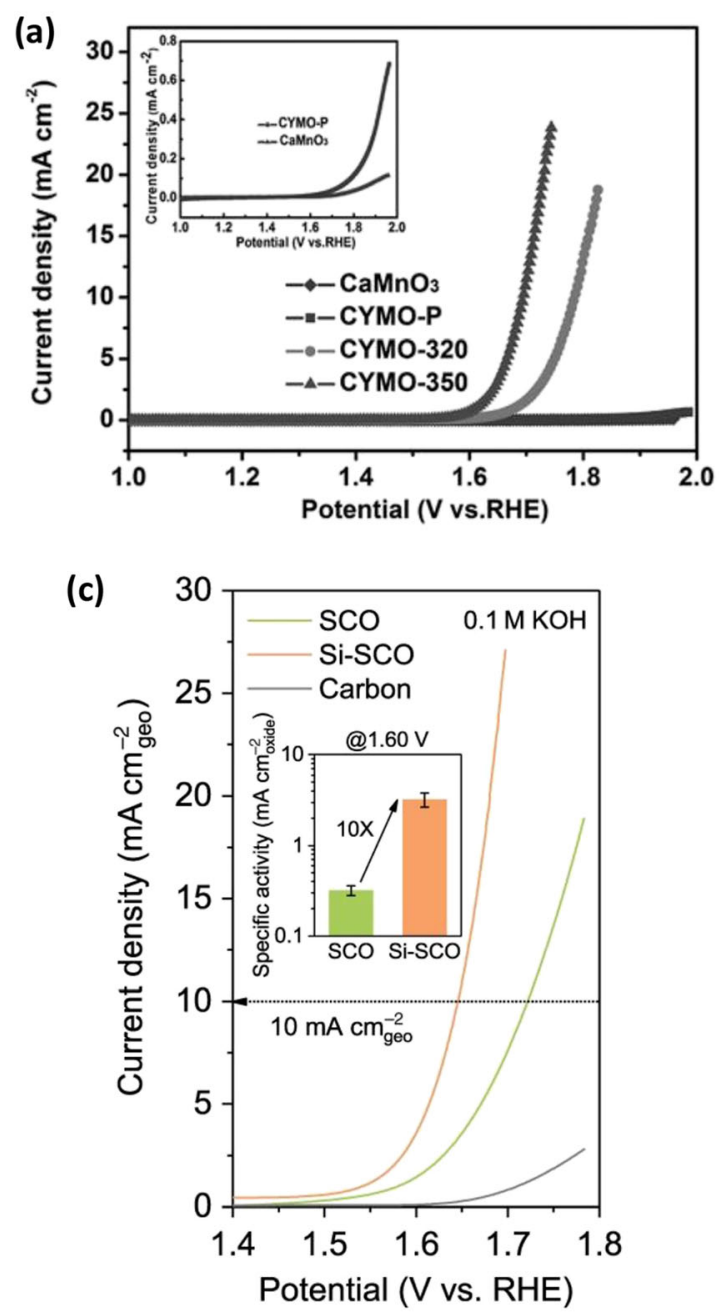

Fig. 9 (a) IR-corrected OER polarization curves, and (b) the OER activity increased with conductivity and $\mathrm{e}_{\mathrm{g}}$ electron-filling status optimized by hydrogen treatment. Inset: Jahn-Teller distortion promoted the formation of oxygen defects, resulting in an optimal $\mathrm{Mn} \mathrm{e}_{\mathrm{g}}$ filling state and better electrical conductivity. Reproduced from Ref. [36] with permission from WILEY-VCH Verlag GmbH \& Co. KGaA, Weinheim, Copyright 2015. (c) OER kinetic currents (normalized to the geometric surface area of the electrode, in $\mathrm{mA} \mathrm{cm}^{-2}$ geo ) of $\mathrm{SCO}$ and $\mathrm{Si}-\mathrm{SCO}$ collected in an $\mathrm{O}_{2}$-saturated $0.1 \mathrm{M} \mathrm{KOH}$ electrolyte under ambient conditions. The contribution

$\left(\mathrm{La}_{0.2} \mathrm{Sr}_{0.8} \mathrm{CoO}_{3}\right)$, congruent to progressive alignment of the $\mathrm{Co}-\mathrm{O}-\mathrm{Co}$ bonds and an oxidation state of Co greater than $\mathrm{Co}^{3+}$.

Density functional theory (DFT) was utilized in this work to calculate the $\mathrm{Co}-\mathrm{O}-\mathrm{Co}$ bond alignment. Figure 10c relates the structure type and Co-O-Co bond alignment to the $\mathrm{Sr}$ doping content. Highest conductivity (300-time increase compared to the pristine undoped $\mathrm{LaCoO}_{3}$ sample), transition to a cubic structure, second highest oxygen vacancy content, and lowest OER overpotential corresponded to the $\mathrm{La}_{0.4} \mathrm{Sr}_{0.6} \mathrm{CoO}_{3}$ sample. This indicates the favorability of cubic $\mathrm{Pm}-3 m$ structures for intrinsic activity towards OER. Interestingly, Sunarso and Zhou dedicated an investigation to structural changes in $\mathrm{LaNiO}_{3-\delta}(\mathrm{LN})$ [104]. In their work, XPS analysis of oxygen (b)

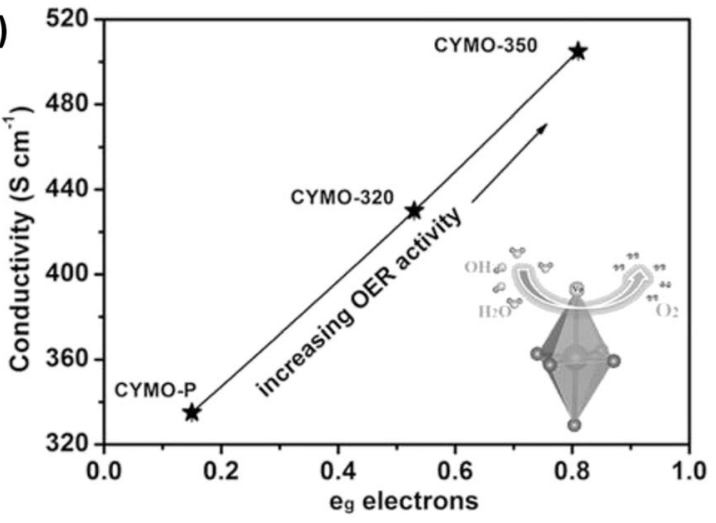

(d)

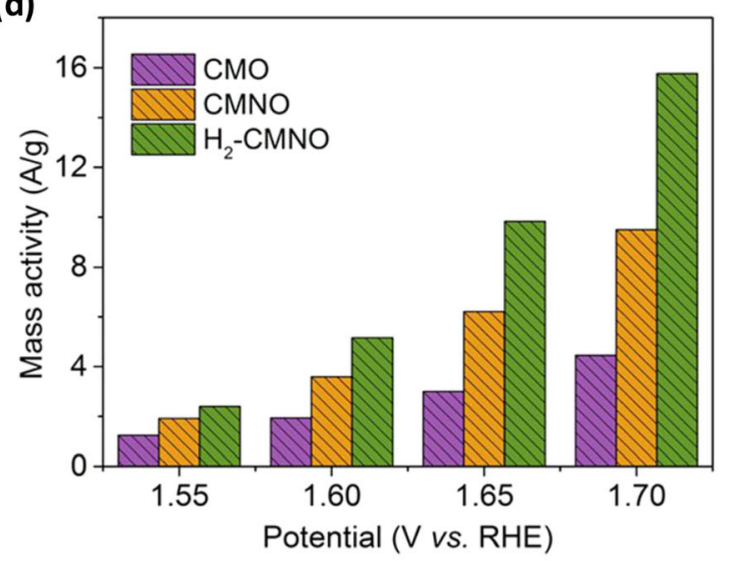

from the conductive carbon as catalyst support is shown for reference. Inset shows the OER-specific activity (normalized to the BET surface area of the oxide catalyst, in $\mathrm{mA} \mathrm{cm}{ }^{-2}$ oxide) of SCO and $\mathrm{Si}-\mathrm{SCO}$ at $1.60 \mathrm{~V}$ vs. RHE. Error bars are the standard deviations of triplicate measurements. Reproduced from Ref. [48] with permission from Springer Nature, Copyright 2020. (d) The mass activity of CMO, CMNO, and $\mathrm{H}_{2}$-CMNO in $\mathrm{O}_{2}$-saturated $0.1 \mathrm{M} \mathrm{KOH}$ solution at $1600 \mathrm{rpm}$. Reproduced from Ref. [45] with permission from the American Chemical Society, Copyright 2017

deficiencies was not undertaken - however, this leaves room for doping, which introduces oxygen vacancies and enhances performance as discussed earlier. Nevertheless, in this early work by the group, a direct relationship between OER/ORR activity and LN structure was identified. LN samples that were heated at 400,600 , and $800{ }^{\circ} \mathrm{C}$ yielded an apparent transition from rhombohedral $(R-3 c)$ to cubic $(P m-3 m)$ and showed a direct correlation with respect to OER/ORR activities - see Fig. 10d. Furthermore, the elongation of the Ni-O bond in the cubic structure favors higher OER/ORR activity.

Computational analyses using varying density functional theory (DFT) approaches have proven invaluable in the investigations of surface microengineering effects including, but not limited to, effects of oxygen vacancies in perovskites. 
(a)

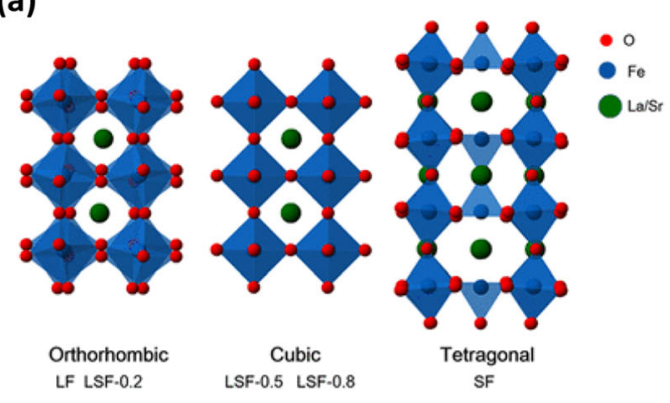

(c)

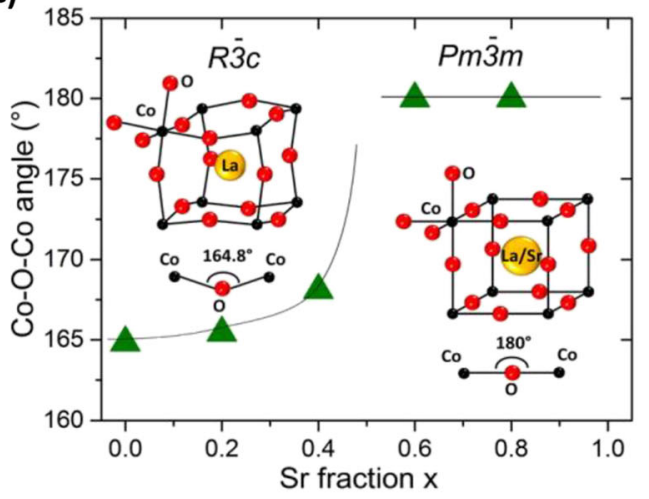

(b)

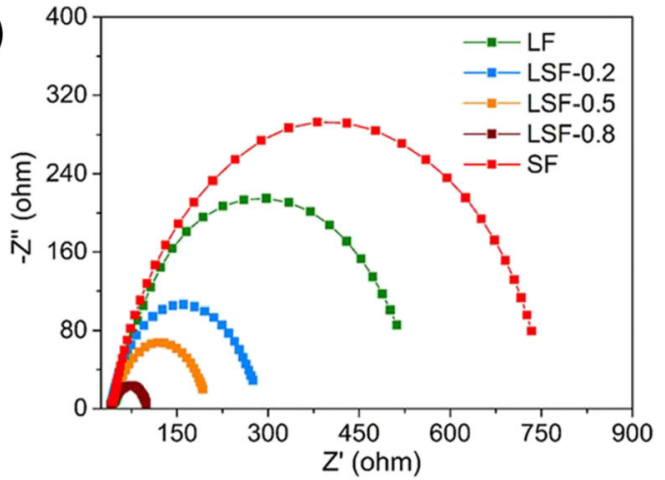

(d)

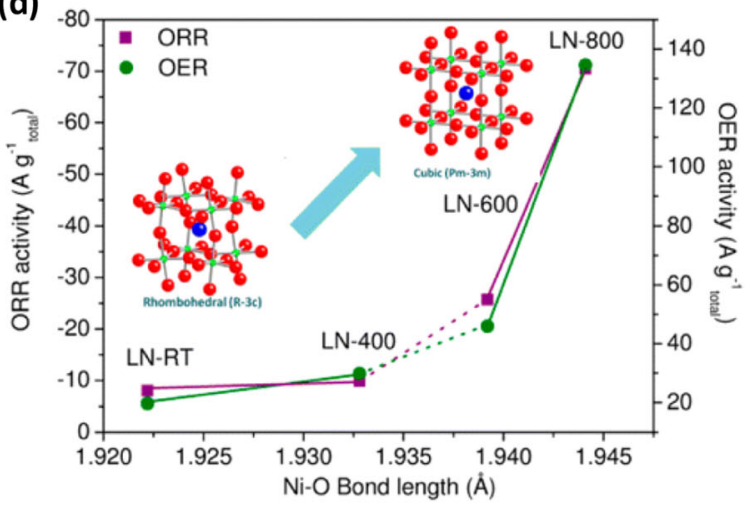

LSC. Reproduced from Ref. [103] with permission from the American Chemical society, Copyright 2015. (d) Relation of ORR/OER with crystallographic structure of LN samples. Reproduced from Ref. [104] with permission from the American Chemical Society, Copyright 2013
The Kohn-Sham scheme is perhaps the most utilized "basecase" method used in DFT. The derivation of the noninteracting and spin density functional respective systems are integrated within the Kohn-Sham scheme. The scheme's equation (Eq. (6)) revolves around motion calculation of interacting electrons as individual moving particles [105]. $\nu_{\mathrm{KS}}(\mathrm{r})$ represents the Kohn-Sham potential, $\mu_{\mathrm{i}}$ shows the one-electron orbital, and $\varepsilon_{\mathrm{i}}$ represents the total energy of one orbital.

$\left[-\frac{1}{2} \nabla^{2}+\nu_{K S}(r)\right] \mu_{i}=\varepsilon_{i} \mu_{i}$

The different intermediates partaking in OER for perovskites and other material types have been heavily investigated using DFT. Ideally, OER intermediates $\left(\mathrm{O}^{*}, \mathrm{OH}^{*}\right.$, and $\left.\mathrm{OOH}^{*}\right)$ should not bind too strongly to the active site of a perovskite, to facilitate for reasonable dynamics. Man et al. utilized this understanding to develop and present a universal scaling relationship between the free binding energy of $\mathrm{OOH}^{*}$ and $\mathrm{OH}^{*}$ in order to analyze the reaction free energy diagrams of all oxides in a general way [106]. This work gave rise to a simple and effective universal descriptor for OER reactions on oxide surfaces, including perovskites, and to an activity volcano plot. At the time, a few materials were at the apex of this activity volcano, representing a theoretical minimum overpotential, whilst remaining materials are burdened by either too weak or too strong $\mathrm{O} *$ intermediate binding energies. The activity descriptor $\left(\Delta \mathrm{G}_{\mathrm{O}^{*}}-\Delta \mathrm{G}_{\mathrm{OH}^{*}}\right)$ remains invaluable in all first principle design of materials, including oxygen-deficient perovskites for the OER. DFT approaches, such as DFT + U, which introduces an artificial corrective potential, are heavily used to study, fine-tune, and guide experimental work for improving OER performance in perovskites. For instance, Yao et al. employed DFT $+\mathrm{U}$ to investigate oxygendeficient $\mathrm{Mg}$-based $\mathrm{A}_{2} \mathrm{Mn}_{2} \mathrm{O}_{5}(\mathrm{~A}=\mathrm{Ca}, \mathrm{Sr})$ [74]. They predicted $\mathrm{Sr}_{2} \mathrm{Mn}_{2} \mathrm{O}_{5}$ to have lower OER overpotentials due to the large ionic radii of A-site elements caused by weakening the hybridization between the $\mathrm{Mn} \mathrm{d}_{\mathrm{z} 2}$ and $\mathrm{OH}-\mathrm{p}_{\sigma}$ orbitals. Furthermore, Zhao et al. computed the Gibbs free energy changes for the OER using pristine $\mathrm{PrBaCo}_{2} \mathrm{O}_{5+\delta}$ ( $\mathrm{PBC}$, where $\mathrm{Co}$ as active center), $\mathrm{PrBa}_{0.5} \mathrm{Sr}_{0.5} \mathrm{Co}_{1.5} \mathrm{Fe}_{0.5} \mathrm{O}_{5+\delta}$ ( $\mathrm{PBSCF}$, where $\mathrm{Co}$ and $\mathrm{Fe}$ as active centers) [101]. Figure 11a shows how the undertaking of the reaction on the Fe active site of modified PBSCF resulted in preferable theoretical thermodynamic overpotentials. Cheng et al. studied the effects of Co-O-Co bond alignment and Srdoping amount (x) in $\mathrm{La}_{1-} \mathrm{Sr}_{\mathrm{x}} \mathrm{CoO}_{3}$ (LSC) [103]. Shin et al. recently used first principles DFT calculations to probe how the hole compensations by electrons released from oxygen vacancies 
(a)

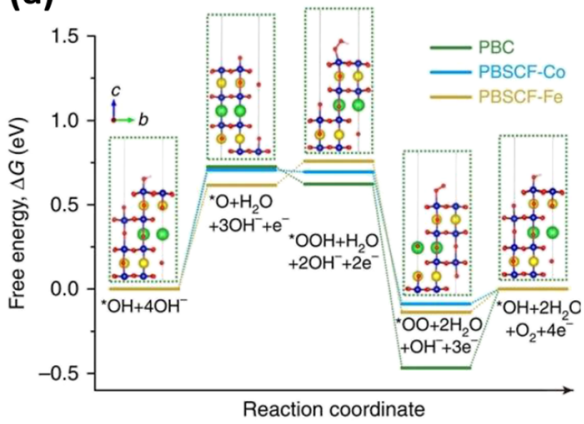

(b)

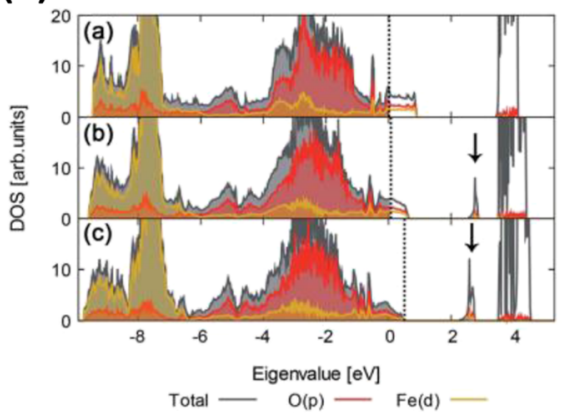

(c)

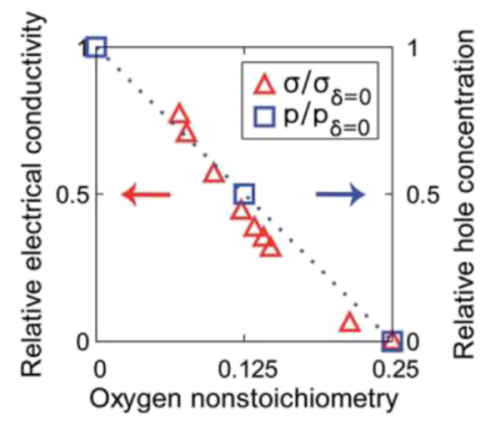

Fig. 11 (a) The computed Gibbs free energy changes for the whole system for the OER at $\eta=0 \mathrm{~V} / 298.15 \mathrm{~K}$ on a $\mathrm{PrBaCo}_{2} \mathrm{O}_{5+\delta}(\mathrm{PBC}) /$ $\mathrm{PrBa}_{0.5} \mathrm{Sr}_{0.5} \mathrm{Co}_{1.5} \mathrm{Fe}_{0.5} \mathrm{O}_{5+\delta}$ (PBSCF) surface; the B-site in PBC (Co) and PBSCF $(\mathrm{Co} / \mathrm{Fe})$ is treated as the catalytically active centers, all adsorbates are bonded to these redox centers; insets are atomic structures of PBC slabs with adsorbates. In all schematics, each deep blue ball is Co or $\mathrm{Fe}$, fluorescent yellow ball is $\mathrm{Pr}$, green ball is $\mathrm{Ba}$ or $\mathrm{Sr}$, red ball is $\mathrm{O}$, and light pink ball is H. Reproduced from Ref. [101] with permission from Springer Nature, Copyright 2017. (b) Comparison of electronic density of states (DOS) for the 2nd structure of (a) $\mathrm{La}_{0.5} \mathrm{Sr}_{0.5} \mathrm{FeO}_{3}$, (b) $\mathrm{La}_{0.5} \mathrm{Sr}_{0.5} \mathrm{FeO}_{2.875}$, and (c) $\mathrm{La}_{0.5}-\mathrm{Sr}_{0.5} \mathrm{FeO}_{2.750}$. Black lines, total DOS; red lines, projected DOS for O; gold lines, projected DOS for Fe. Filled (empty) regions, occupied (unoccupied) states; vertical dotted lines, valence band maxima; black arrows, oxygen vacancy states. (c) Relative electrical conductivity ( $\mathrm{s} / \mathrm{sd}^{1} / 40$, red triangle) and relative hole concentration ( $\mathrm{p} / \mathrm{pd}^{1} / 40$, blue square) as a function of oxygen non-stoichiometry, with values at stoichiometric state $\left(\mathrm{d}^{1} / 40\right)$ as reference state. Blue dotted line, trend of the hole concentration. Electrical conductivity is obtained from the experimental data [107], and hole concentration is estimated by integrating the unoccupied state density of the hybridized band. Reproduced from Ref. [108] with permission from The Royal Chemical Society, Copyrights 2020 in $\mathrm{La}_{0.5} \mathrm{Sr}_{0.5} \mathrm{FeO}_{3-\delta}$ eventually lowers the conductivity and leads to metal-to-semiconductor transition at low partial pressures of $\mathrm{O}_{2}$ [108]. Density of states (DOS) calculations in Fig. 11b show how the Fermi level, initially in the middle of the hybridization band for $\mathrm{O} 2 \mathrm{p}$ and $\mathrm{Fe} 3 \mathrm{~d}$ orbitals, increases. Two oxygen vacancy states appear, and as a result, the hybridized band becomes fully occupied. The excess electrons from the vacancies thus increase the Fermi level and cause the transition from metallic to semiconductor state, in this case leading to a decrease in conductivity (Fig. 11c). DFT under the general gradient approach (GGA), such as the Perdew-Burke-Ernzerhof (PBE) method, has proven to be an effective strategy for initial screening of oxygendeficient materials for electrolysis applications [109]. However, there are still opportunities for improvement by including orbital dependent potentials and hybrid functionals (i.e., HSE06, PBE0) that can provide more accurate electronics analysis (i.e., band gaps for semiconductors, band structure) compared with the basic PBE approach at the cost of high computational resources $[110,111]$. Incorporation of relatively recent methods, such as the GW approximation for calculating self-energy of a main body, is highly expected to curb limiting computational resources especially for large systems [112].

\section{Disadvantageous effects of oxygen vacancies in perovskites}

The previous section discussed changes in the intrinsic effects that promote an enhanced OER performance as perovskites become oxygen deficient to some degree. However, we will now focus on cases which do not fall under this general category.
Several reports have discussed important intrinsic characteristics - electric conductivity, phase transformations, stability - that are disadvantageously affected upon significant reduction of a perovskite. Recently, Yamada et al. reported phase transformation and relationship of structure with stability for different degrees of oxygen deficiencies $(\delta)$ in $\mathrm{Ba}_{0.5} \mathrm{Sr}_{0.5} \mathrm{Co}_{0.8} \mathrm{Fe}_{0.2} \mathrm{O}_{3-\delta}$ (BSCF) perovskite oxides [95]. OER activity was evaluated for a range of oxygen-deficient BSCF perovskites, namely heavily oxygen vacant $(\mathrm{r}-\mathrm{BSCF}, \delta=0.51$ ), pristine conventional ( $\mathrm{p}$-BSCF, $\delta=0.38$ ), and highly oxidized (o-BSCF, $\delta=0.12$ ) BSCF. Interestingly, overpotentials for heavily oxygen vacant and highly oxidized samples were inferior to that of the moderate and conventional sample $(\delta=0.38)$. Figure 12a reports the lattice parameter (a) increases linearly with respect to the degree of oxygen vacancy $(\delta)$ in BSCF, indicating an increase in the ionic radii of the $\mathrm{B}$-site ions $(\mathrm{Fe}, \mathrm{Co})$ which causes structural instabilities $[114,115]$. Figure $12 \mathrm{~b}$ shows the relationship between $\delta$ and the recorded OER overpotential. Moreover, Du et al. reported excessive reduction via annealing in $\mathrm{H}_{2}$-reducing atmosphere led to the phase transformation of $\mathrm{CaMnO}_{3}$ from Pnma to Pbam $\left(\mathrm{CaMnO}_{2.5}\right)$ [113]. The oxygendeficient and stable $\mathrm{CaMnO}_{2.75}$ sample - showing the lower OER overpotential - was experimentally found to be the most conductive $\left(4.68 \times 10^{-2} \mathrm{~S} \mathrm{~m}^{-2}\right)$ compared with that of pristine $\mathrm{CaMnO}_{3}\left(1.56 \times 10^{-3} \mathrm{~S} \mathrm{~m}^{-2}\right)$ and $\mathrm{CaMnO}_{2.5}\left(1.8 \times 10^{-3} \mathrm{~S} \mathrm{~m}^{-2}\right)$.

In the cases of $\mathrm{BSCF}$ and $\mathrm{CaMnO}_{2}$ perovskites discussed previously, a certain degree of vacancies is favorable, but this is not necessary the case for the wide variety of perovskite oxides. For instance, Hirai et al. showed that the OER activity of layered perovskite $\mathrm{Sr}_{2} \mathrm{VFeAsO}_{3-\delta}$ was substantially improved above lattice oxygen vacancy content of $\delta=0.5$, 
Fig. 12 (a) Lattice constant (a) as a function of oxygen deficiency $(\delta)$ for BSCF. The line represents the least square fitting result and (b) OER overpotential $(\eta)$ for $\mathrm{p}$ BSCF, r-BSCF, and o-BSCF. Reproduced from Ref. [95] with permission from The Royal Chemical Society, Copyright 2019. (c) DFT calculation of the density of states (DOS) of pristine $\mathrm{CaMnO}_{3}, \mathrm{CaMnO}_{2.75}$, and $\mathrm{CaMnO}_{2.5}$. Reproduced from Ref. [113] with permission from the American Chemical Society, Copyright 2014. (d) Polarization curves for OER activities of double perovskite $\mathrm{PrBaCo}_{2} \mathrm{O}_{6-\delta}$ in $\mathrm{O}_{2}$-saturated $1.0 \mathrm{M} \mathrm{KOH}$. Reproduced from Ref. [38] with permission from The Royal Chemical Society, Copyright 2019 (a)

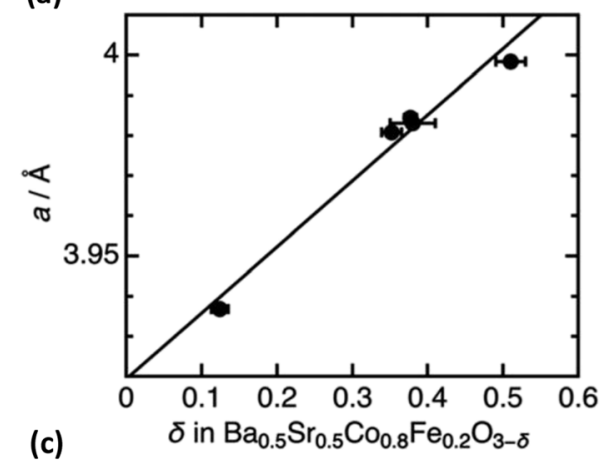

(c)

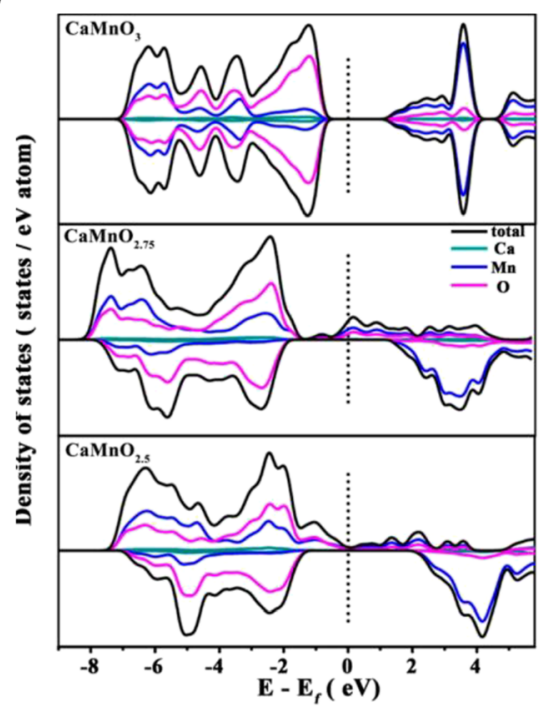

(b)

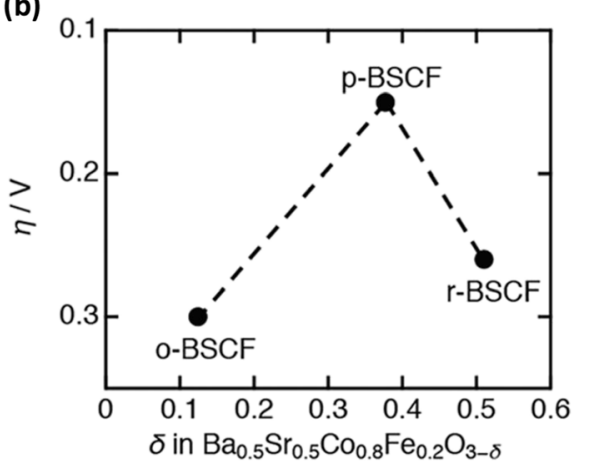

(d)

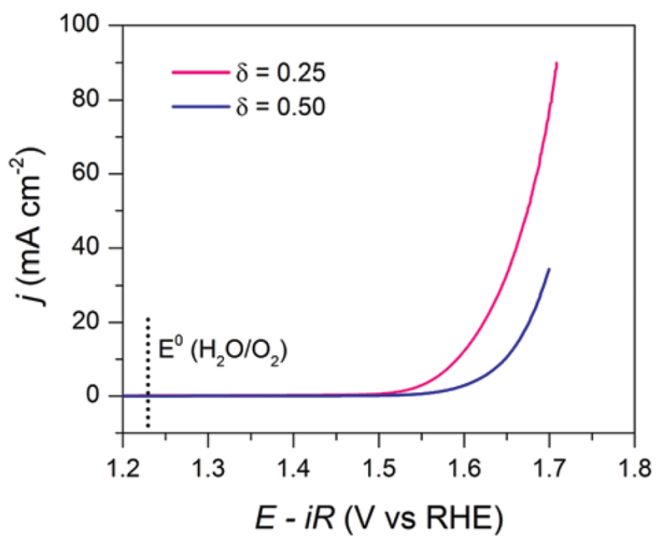

manifesting near $300 \mathrm{mV}$ reduction in overpotential and 80 times higher specific activity at $1.7 \mathrm{~V}$ vs. RHE [116]. Chronoamperometric (CA) tests revealed that formation of oxygen vacancies in $\mathrm{Sr}_{2} \mathrm{VFeAsO}_{3-\delta}$ does not cause structural instabilities. DFT calculations for $\delta>0.5$ show that the oxygen vacant sites become available and promote $\mathrm{OH}^{-}$adsorbates through more favorable $\mathrm{O}-\mathrm{O}$ bond formation, which further lowers enthalpic limitations for the OER.

DFT calculations were employed in order to generate a density of states (DOS) plot shown in Fig. 12c and indicate the creation of new electronic states near the Fermi level, which are known to enhance conductivity and allow favorable binding energies with OER intermediates. Furthermore, the same DOS plot predicts a $1.56 \mathrm{eV}$ bandgap for the pristine perovskite sample, indicating the expected semiconductor behavior. The creation of states in only spin-up near the Fermi level in the DOS of $\mathrm{CaMnO}_{2.75}$ and an apparent gap for the spin-down states indicate a highly spin polarized system exhibiting metallicity, which enhances conductivity. Miao et al. explored the role of oxygen vacancies in the OER of double perovskite $\mathrm{PrBaCo}_{2} \mathrm{O}_{6-\delta}$ [38]. Confirming previous reports of the disadvantageous effects excessive oxygen vacancies can cause for OER performance of perovskites, the authors found that intrinsic activity decreases for this cobalt perovskite. Oxygen vacancies were found to form an ordered alignment of $\mathrm{PrO}_{1-\delta}$ layers with an alternative configuration of pyramidal $\mathrm{Co}^{3+} \mathrm{O}_{5}$ and octahedral $\mathrm{Co}^{3+} \mathrm{O}_{6}$, resulting in spin state transition of $\mathrm{Co}^{3+}$ ions in the tetrahedral formation from high-spin (HS: $\mathrm{t}^{4}{ }_{2 \mathrm{~g}} \mathrm{e}^{2}$ ) to low-spin (LS: $\mathrm{t}^{5}{ }_{2 \mathrm{~g}} \mathrm{e}_{\mathrm{g}}$ ) states. This transition follows a reduction in the $\mathrm{e}_{\mathrm{g}}$ occupancy away from ideal unity and a decrease in the conductivity. Furthermore, an apparent weakening in the $\mathrm{Co}-\mathrm{O}$ bond covalency results in higher instabilities and reduction in the alkaline $(1 \mathrm{M} \mathrm{KOH})$ OER performance. Deficiencies in the $\mathrm{Co}-\mathrm{O}$ bond covalency in $\mathrm{ABO}_{3}$-type oxides caused by lattice distortions can severely limit electrochemical performance [117]. Figure 12d shows that as the oxygen vacancy content $(\delta)$ increases from 0.25 to 0.50 , both the overpotential at $10 \mathrm{~mA} \mathrm{~cm}^{-2}$ increases from 360 to $420 \mathrm{mV}$ and a corresponding increase in the Tafel slopes from 70 to $80 \mathrm{mV} \mathrm{dec}{ }^{-1}$, respectively.

\section{Conclusion and future outlook}

This review gives an executive, yet thorough, examination in the development of oxygen-deficient perovskites. A discussion pertaining to the major contributing factors of performance enhancements through electronic modulation has been 
undertaken. Attaining $\mathrm{e}_{\mathrm{g}}$ occupancy close to unity, minimizing $\Delta \mathrm{E}_{\mathrm{d}-\mathrm{p}}$, enhancing conductivity, and microengineering the surface for optimum and moderate binding energies for the OER intermediates $(\mathrm{O}, \mathrm{OH}, \mathrm{OOH})$ via the induction of an oxygen-deficient perovskite surface has been presented. The importance of careful tuning of oxygen vacancies in order to prevent structural instabilities was discussed.

Further research is needed in order to enhance the stability of perovskites generally, which are known to undergo amorphization during OER operations through the leaching of $\mathrm{A} / \mathrm{B}$-site cations, as is the case with BSCF materials [118]. On that note, propagated investigation of highly oxygen-deficient layered perovskites is encouraged, due to their innate stabilities at high $\delta$ values and oxygen vacancy oriented OER mechanism. The apparent lower stabilities exhibited in perovskites for longterm OER operation compared with $\mathrm{Ni}$ - and Fe-based materials are hindering some interest in them. However, this presents a great area of potential development for these materials. Furthermore, the relatively costly and multi-process steps involved in the fabrication and fine-tuning of oxygen vacancies in oxygen-deficient perovskites presently bottlenecks their potential utilization for large scale applications. Development of more environmentally benign and one-step syntheses for perovskites is expected to revolutionize development and applications of these electrocatalysts. The modification of perovskites through other types of defects and/or heterojunction with novel supports, such as conductive MXenes, may prove fruitful in enhancing their electrocatalytic performance.

The utilization of computational techniques, such as DFT, to accurately screen and predict favorable and highly active perovskite surfaces is of invaluable importance. DFT employment to further explore and understand the reaction mechanisms on perovskite surface during OER will lead to more rationally designed materials with more surface functionalities and properties. However, consideration should be taken when purely relying on DFT analysis for predicting performance of TMO electrocatalysts. Currently, functionals and pseudopotentials within conventional DFT can predict well the performance of $3 \mathrm{~d}$ metals, but they do not work well for certain $4 \mathrm{~d}$ and 5d TMOs (e.g., $\mathrm{Ca}_{1-\mathrm{x}} \mathrm{Sr}_{\mathrm{x}} \mathrm{RuO}_{3}, \mathrm{SrIrO}_{3}$ ) [119, 120]. In such systems, direct observation of the electronic structure (bulk-sensitive photoelectron spectroscopy) are conducted to understand their electronic structure. Furthermore, implementation of machine learning (ML) approaches for quick prediction and selection of effective catalytic surfaces has been on the rise [121]. For instance, $\mathrm{Li}$ et al. have recently presented an adaptive ML framework for OER perovskites, which navigated through numerous potential double perovskites $\left(\mathrm{AA}^{\prime} \mathrm{B}_{2} \mathrm{O}_{6}\right)$ and identified feasible surfaces for fabrication [80, 113]. Appropriating ML techniques to identify the most prominent activity descriptors on surface-modulated perovskites will surely lead to a high-throughput propagation of nextgeneration perovskite electrocatalysts.
We believe that it is of paramount importance to rationalize the design of perovskites, and electrocatalytic materials in general, for an effective and feasible integration of such materials as viable industrial electrocatalysts in water splitting. Tuning activity descriptors relevant to the performance of oxygen-deficient perovskite electro-anodes, coupled with recent synthesis techniques for surface-modulated structures, offer potential for further improvement of catalytic activity. Bridging the gap between DFT, ML, and experimental work has been highlighted due to the immense potential it brings towards efficient and systematic approach for discovery of highly active and stable catalysts.

Acknowledgments This study was made possible by a grant from the Qatar National Research Fund under its National Priorities Research Program award number NPRP12S-0131-190024. The paper's contents are solely the responsibility of the authors and do not necessarily represent the official views of the Qatar National Research Fund.

Funding Open Access funding provided by the Qatar National Library.

\section{Compliance with ethical standards}

Conflict of interest The authors declare that there is no conflict of interest involved in this submission.

Open Access This article is licensed under a Creative Commons Attribution 4.0 International License, which permits use, sharing, adaptation, distribution and reproduction in any medium or format, as long as you give appropriate credit to the original author(s) and the source, provide a link to the Creative Commons licence, and indicate if changes were made. The images or other third party material in this article are included in the article's Creative Commons licence, unless indicated otherwise in a credit line to the material. If material is not included in the article's Creative Commons licence and your intended use is not permitted by statutory regulation or exceeds the permitted use, you will need to obtain permission directly from the copyright holder. To view a copy of this licence, visit http://creativecommons.org/licenses/by/4.0/.

\section{References}

1. J. Wang, Y. Gao, D. Chen, J. Liu, Z. Zhang, Z. Shao, F. Ciucci, Water splitting with an enhanced bifunctional double perovskite. ACS Catal. 8, 364-371 (2018)

2. Y. Yan, B.Y. Xia, B. Zhao, X. Wang, A review on noble-metalfree bifunctional heterogeneous catalysts for overall electrochemical water splitting. J. Mater. Chem. A 4, 17587-17603 (2016)

3. H. Wang, M. Zhou, P. Choudhury, H. Luo, Perovskite oxides as bifunctional oxygen electrocatalysts for oxygen evolution/ reduction reactions - a mini review. Appl. Mater. Today 16, 5671 (2019)

4. J. Turner, G. Sverdrup, M.K. Mann, P.C. Maness, B. Kroposki, M. Ghirardi, R.J. Evans, D. Blake, Renewable hydrogen production. Int. J. Energy Res. 32, 379-407 (2008)

5. C. Hu, L. Zhang, J. Gong, Recent progress made in the mechanism comprehension and design of electrocatalysts for alkaline water splitting. Energy Environ. Sci. 12, 2620-2645 (2019) 
6. Z. Zong, Z. Qian, Z. Tang, Z. Liu, Y. Tian, S. Wang, Hydrogen evolution and oxygen reduction reactions catalyzed by coreshelled Fe@Ru nanoparticles embedded in porous dodecahedron carbon. J. Alloys Compd. 784, 447-455 (2019)

7. J. Wang, X. Yue, Y. Yang, S. Sirisomboonchai, P. Wang, X. Ma, A. Abudula, G. Guan, Earth-abundant transition-metal-based bifunctional catalysts for overall electrochemical water splitting: a review. J. Alloys Compd. 819, 153346 (2020)

8. P. Ebrahimi, A. Kumar, M. Khraisheh, A review of recent advances in water-gas shift catalysis for hydrogen production. Emergent Mater (2020). https://doi.org/10.1007/s42247-02000116-y

9. L.M. Gandía, R. Oroz, A. Ursúa, P. Sanchis, P.M. Diéguez, Renewable hydrogen production: performance of an alkaline water electrolyzer working under emulated wind conditions. Energy Fuel 21, 1699-1706 (2007)

10. P. Sharma, P. Doiphode, O. Bhorade, A. Yengantiwar, Bismuth vanadate thin films for efficient photoelectrochemical water splitting. Emergent Mater 3, 187-194 (2020)

11. Z. Wu, N. Ambrožová, E. Eftekhari, N. Aravindakshan, W. Wang, Q. Wang, S. Zhang, K. Kočí, Q. Li, Photocatalytic H2 generation from aqueous ammonia solution using $\mathrm{TiO} 2$ nanowires-intercalated reduced graphene oxide composite membrane under low power UV light. Emergent Mater 2, 303-311 (2019)

12. J. Song, C. Wei, Z.-F. Huang, C. Liu, L. Zeng, X. Wang, Z.J. Xu, A review on fundamentals for designing oxygen evolution electrocatalysts. Chem. Soc. Rev. 49, 2196-2214 (2020)

13. Y. Guo, T. Park, J.W. Yi, J. Henzie, J. Kim, Z. Wang, B. Jiang, Y. Bando, Y. Sugahara, J. Tang, Nanoarchitectonics for transitionmetal-sulfide-based electrocatalysts for water splitting. Adv. Mater. 31, 1807134 (2019)

14. J. Joo, T. Kim, J. Lee, S.I. Choi, K. Lee, Morphology-controlled metal sulfides and phosphides for electrochemical water splitting. Adv. Mater. 31, 1806682 (2019)

15. J.O.M. Bockris, T. Otagawa, Mechanism of oxygen evolution on perovskites. J. Phys. Chem. 87, 2960-2971 (1983)

16. X. Li, X. Hao, A. Abudula, G. Guan, Nanostructured catalysts for electrochemical water splitting: current state and prospects. J. Mater. Chem. A 4, 11973-12000 (2016)

17. F. Song, L. Bai, A. Moysiadou, S. Lee, C. Hu, L. Liardet, X. Hu, Transition metal oxides as electrocatalysts for the oxygen evolution reaction in alkaline solutions: an application-inspired renaissance. J. Am. Chem. Soc. 140, 7748-7759 (2018)

18. Y. Matsumoto, E. Sato, Electrocatalytic properties of transition metal oxides for oxygen evolution reaction. Mater. Chem. Phys. 14, 397-426 (1986)

19. E. Fabbri, A. Habereder, K. Waltar, R. Kötz, T.J. Schmidt, Developments and perspectives of oxide-based catalysts for the oxygen evolution reaction. Catalysis Sci. Technol. 4, 3800-3821 (2014)

20. M.Q. Yang, J. Wang, H. Wu, G.W. Ho, Noble metal-free nanocatalysts with vacancies for electrochemical water splitting. Small 14, 1703323 (2018)

21. Z.W. Seh, J. Kibsgaard, C.F. Dickens, I. Chorkendorff, J.K. Nørskov, T.F. Jaramillo, Combining theory and experiment in electrocatalysis: Insights into materials design. Science $\mathbf{3 5 5}$, eaad4998 (2017)

22. M. Escudero-Escribano, A.F. Pedersen, E.A. Paoli, R. Frydendal, D. Friebel, P. Malacrida, J. Rossmeisl, I.E.L. Stephens, I. Chorkendorff, Importance of surface IrOx in stabilizing $\mathrm{RuO} 2$ for oxygen evolution. J. Phys. Chem. B 122, 947-955 (2018)

23. H. Cao, X. Gong, T. Liu, F. Xiao, X. Lv, J. Zhou, L. Gai, Hierarchical Co-N microballs with heterostructure exhibiting superior electrochemical properties for water splitting and reduction of I3-. J. Alloys Compd. 797, 341-347 (2019)
24. J. Bao, X. Zhang, B. Fan, J. Zhang, M. Zhou, W. Yang, X. Hu, H. Wang, B. Pan, Y. Xie, Ultrathin spinel-structured nanosheets rich in oxygen deficiencies for enhanced electrocatalytic water oxidation. Angew. Chem. Int. Ed. 54, 7399-7404 (2015)

25. S. Malkhandi, B. Yang, A.K. Manohar, A. Manivannan, G.K.S. Prakash, S.R. Narayanan, Electrocatalytic properties of nanocrystalline calcium-doped lanthanum cobalt oxide for bifunctional oxygen electrodes. J. Phys. Chem. Lett. 3, 967-972 (2012)

26. J.B. Goodenough, Electronic and ionic transport properties and other physical aspects of perovskites. Rep. Prog. Phys. 67, 1915-1915 (2004) 1993

27. X. Xu, W. Wang, W. Zhou, Z. Shao, Recent advances in novel nanostructuring methods of perovskite electrocatalysts for energyrelated applications. Small Methods 2, 1800071-1800071 (2018)

28. I. Hussain, H.P. Tran, J. Jaksik, J. Moore, N. Islam, M.J. Uddin, Functional materials, device architecture, and flexibility of perovskite solar cell. Emergent Mater 1, 133-154 (2018)

29. O. Malinkiewicz, M. Imaizumi, S.B. Sapkota, T. Ohshima, S. Öz, Radiation effects on the performance of flexible perovskite solar cells for space applications. Emergent Mater 3, 9-14 (2020)

30. C. Sun, R. Hui, J. Roller, Cathode materials for solid oxide fuel cells: a review. J. Solid State Electrochem. 14, 1125-1144 (2010)

31. E. Zhao, Z. Jia, L. Zhao, Y. Xiong, C. Sun, M.E. Brito, One dimensional $\mathrm{La} 0.8 \mathrm{Sr} 0.2 \mathrm{Co} 0.2 \mathrm{Fe} 0.8 \mathrm{O} 3-\delta / \mathrm{Ce} 0.8 \mathrm{Gd} 0.2 \mathrm{O} 1.9$ nanocomposite cathodes for intermediate temperature solid oxide fuel cells. J. Power Sources 219, 133-139 (2012)

32. W. Yang, T. Hong, S. Li, Z. Ma, C. Sun, C. Xia, L. Chen, Perovskite $\mathrm{Sr} 1-\mathrm{xCexCoO} 3-\delta(0.05 \leq \mathrm{x} \leq 0.15)$ as superior cathodes for intermediate temperature solid oxide fuel cells. ACS Appl. Mater. Interfaces 5, 1143-1148 (2013)

33. Q. Ji, L. Bi, J. Zhang, H. Cao, X.S. Zhao, The role of oxygen vacancies of $\mathrm{ABO} 3$ perovskite oxides in the oxygen reduction reaction. Energy Environ. Sci. 13, 1408-1428 (2020)

34. N.-T. Suen, S.-F. Hung, Q. Quan, N. Zhang, Y.-J. Xu, H.M. Chen, Electrocatalysis for the oxygen evolution reaction: recent development and future perspectives. Chem. Soc. Rev. 46, 337-365 (2017)

35. C. Jin, X. Cao, L. Zhang, C. Zhang, R. Yang, Preparation and electrochemical properties of urchin-like $\mathrm{La} 0.8 \mathrm{Sr} 0.2 \mathrm{MnO} 3$ perovskite oxide as a bifunctional catalyst for oxygen reduction and oxygen evolution reaction. J. Power Sources 241, 225-230 (2013)

36. Y. Guo, Y. Tong, P. Chen, K. Xu, J. Zhao, Y. Lin, W. Chu, Z. Peng, C. Wu, Y. Xie, Engineering the electronic state of a perovskite electrocatalyst for synergistically enhanced oxygen evolution reaction. Adv. Mater. 27, 5989-5994 (2015)

37. K. Lam, Y. Gao, J. Wang, F. Ciucci, H2O2 treated $\mathrm{La} 0.8 \mathrm{Sr} 0.2 \mathrm{CoO} 3-\delta$ as an efficient catalyst for oxygen evolution reaction. Electrochim. Acta 244, 139-145 (2017)

38. X. Miao, L. Wu, Y. Lin, X. Yuan, J. Zhao, W. Yan, S. Zhou, L. Shi, The role of oxygen vacancies in water oxidation for perovskite cobalt oxide electrocatalysts: are more better? Chem. Commun. 55, 1442-1445 (2019)

39. P. Aversa, S. Öz, E. Jung, O. Plantevin, O. Cavani, N. Ollier, J.-E. Bourée, B. Geffroy, T. Miyasaka, S. Mathur, C. Corbel, Electron irradiation induced aging effects on radiative recombination properties of quadruple cation organic-inorganic perovskite layers. Emergent Mater 3, 133-160 (2020)

40. J. Kim, X. Yin, K.C. Tsao, S. Fang, H. Yang, Ca2Mn2O5 as oxygen-deficient perovskite electrocatalyst for oxygen evolution reaction. J. Am. Chem. Soc. 136, 14646-14649 (2014)

41. S. She, J. Yu, W. Tang, Y. Zhu, Y. Chen, J. Sunarso, W. Zhou, Z. Shao, Systematic study of oxygen evolution activity and stability on $\mathrm{La} 1-\mathrm{xSrxFeO} 3-\delta$ perovskite electrocatalysts in alkaline media. ACS Appl. Mater. Interfaces 10, 11715-11721 (2018) 
42. J.T. Mefford, X. Rong, A.M. Abakumov, W.G. Hardin, S. Dai, A.M. Kolpak, K.P. Johnston, K.J. Stevenson, Water electrolysis on La1-xSrxCoO3- $\delta$ perovskite electrocatalysts. Nat. Commun. 7, $1-11(2016)$

43. S.S. Pramana, A. Cavallaro, C. Li, A.D. Handoko, K.W. Chan, R.J. Walker, A. Regoutz, J.S. Herrin, B.S. Yeo, D.J. Payne, J.A. Kilner, M.P. Ryan, S.J. Skinner, Crystal structure and surface characteristics of Sr-doped GdBaCo2O6-: $\delta$ double perovskites: oxygen evolution reaction and conductivity. J. Mater. Chem. A 6, 5335-5345 (2018)

44. K. Zhu, T. Wu, M. Li, R. Lu, X. Zhu, W. Yang, Perovskites decorated with oxygen vacancies and $\mathrm{Fe}-\mathrm{Ni}$ alloy nanoparticles as high-efficiency electrocatalysts for the oxygen evolution reaction. J. Mater. Chem. A 5, 19836-19845 (2017)

45. Y.Q. Lyu, F. Ciucci, Activating the bifunctionality of a perovskite oxide toward oxygen reduction and oxygen evolution reactions. ACS Appl. Mater. Interfaces 9, 35829-35836 (2017)

46. D. Chen, J. Wang, Z. Zhang, Z. Shao, F. Ciucci, Boosting oxygen reduction/evolution reaction activities with layered perovskite catalysts. Chem. Commun. 52, 10739-10742 (2016)

47. N.I. Kim, S.H. Cho, S.H. Park, Y.J.Y.J. Lee, R.A. Afzal, J. Yoo, Y.S. Seo, Y.J.Y.J. Lee, J.Y. Park, B-site doping effects of $\mathrm{NdBa} 0.75 \mathrm{Ca} 0.25 \mathrm{Co} 2 \mathrm{O} 5+\delta$ double perovskite catalysts for oxygen evolution and reduction reactions. J. Mater. Chem. A 6, 17807-17818 (2018)

48. Y. Pan, X. Xu, Y. Zhong, L. Ge, Y. Chen, J.-P.M. Veder, D. Guan, R. O'Hayre, M. Li, G. Wang, Direct evidence of boosted oxygen evolution over perovskite by enhanced lattice oxygen participation. Nat. Commun. 11, 1-10 (2020)

49. C.F. Chen, G. King, R.M. Dickerson, P.A. Papin, S. Gupta, W.R. Kellogg, G. Wu, Oxygen-deficient BaTiO3-x perovskite as an efficient bifunctional oxygen electrocatalyst. Nano Energy 13, 423-432 (2015)

50. Y. Zhu, W. Zhou, J. Yu, Y. Chen, M. Liu, Z. Shao, Enhancing electrocatalytic activity of perovskite oxides by tuning cation deficiency for oxygen reduction and evolution reactions. Chem. Mater. 28, 1691-1697 (2016)

51. Y. Zhu, L. Zhang, B. Zhao, H. Chen, X. Liu, R. Zhao, X. Wang, J. Liu, Y. Chen, M. Liu, Improving the activity for oxygen evolution reaction by tailoring oxygen defects in double perovskite oxides. Adv. Funct. Mater. 29, 1901783 (2019)

52. R.A. Rincón, E. Ventosa, F. Tietz, J. Masa, S. Seisel, V. Kuznetsov, W. Schuhmann, Evaluation of perovskites as electrocatalysts for the oxygen evolution reaction. ChemPhysChem 15, 2810-2816 (2014)

53. S. Peng, X. Han, L. Li, S. Chou, D. Ji, H. Huang, Y. Du, J. Liu, S. Ramakrishna, Electronic and defective engineering of electrospun $\mathrm{CaMnO} 3$ nanotubes for enhanced oxygen electrocatalysis in rechargeable zinc-air batteries. Adv. Energy Mater. 8, 1800612 (2018)

54. Y. Lu, A. Ma, Y. Yu, R. Tan, C. Liu, P. Zhang, D. Liu, J. Gui, Engineering oxygen vacancies into $\mathrm{LaCoO} 3$ perovskite for efficient electrocatalytic oxygen evolution. ACS Sustain. Chem. Eng. 7, 2906-2910 (2019)

55. H. Wang, X. Chen, D. Huang, M. Zhou, D. Ding, H. Luo, Cation deficiency tuning of $\mathrm{LaCoO} 3$ perovskite as bifunctional oxygen electrocatalyst. ChemCatChem 12, 2768-2775 (2020)

56. N.I. Kim, Y.J. Sa, T.S. Yoo, S.R. Choi, R.A. Afzal, T. Choi, Y.S. Seo, K.S. Lee, J.Y. Hwang, W.S. Choi, S.H. Joo, J.Y. Park, Oxygen-deficient triple perovskites as highly active and durable bifunctional electrocatalysts for oxygen electrode reactions. Sci. Adv. 4, eaap9360 (2018)

57. D. Guan, G. Ryu, Z. Hu, J. Zhou, C.-L. Dong, Y.-C. Huang, K. Zhang, Y. Zhong, A.C. Komarek, M. Zhu, Utilizing ion leaching effects for achieving high oxygen-evolving performance on hybrid nanocomposite with self-optimized behaviors. Nat. Commun. 11, 1-10 (2020)

58. R.K. Hona, F. Ramezanipour, Remarkable oxygen-evolution activity of a perovskite oxide from the $\mathrm{Ca} 2-\mathrm{x} \mathrm{Sr} x \mathrm{Fe} 2 \mathrm{O} 6-\delta$ series. Angew. Chem. Int. Ed. 58, 2060-2063 (2019)

59. J. Bian, Z. Li, N. Li, C. Sun, Oxygen deficient LaMn0. 75Co0. $2503-\delta$ nanofibers as an efficient electrocatalyst for oxygen evolution reaction and zinc-air batteries. Inorg. Chem. 58, 8208-8214 (2019)

60. J. Bian, R. Su, Y. Yao, J. Wang, J. Zhou, F. Li, Z.L. Wang, C. Sun, $\mathrm{Mg}$ doped perovskite $\mathrm{LaNiO} 3$ nanofibers as an efficient bifunctional catalyst for rechargeable zinc-air batteries. ACS Appl Energy Mater 2, 923-931 (2019)

61. X. Xu, C. Su, W. Zhou, Y. Zhu, Y. Chen, Z. Shao, Co-doping strategy for developing perovskite oxides as highly efficient electrocatalysts for oxygen evolution reaction. Adv Sci 3, 1500187 (2016)

62. J. Suntivich, K.J. May, H.A. Gasteiger, J.B. Goodenough, Y. Shao-Horn, A perovskite oxide optimized for oxygen evolution catalysis from molecular orbital principles. Science 334, 13831385 (2011)

63. S. Gupta, W. Kellogg, H. Xu, X. Liu, J. Cho, G. Wu, Bifunctional perovskite oxide catalysts for oxygen reduction and evolution in alkaline media. Chem. Asian J. 11, 10-21 (2016)

64. D.A. Kuznetsov, B. Han, Y. Yu, R.R. Rao, J. Hwang, Y. RománLeshkov, Y. Shao-Horn, Tuning redox transitions via inductive effect in metal oxides and complexes, and implications in oxygen electrocatalysis. Joule 2, 225-244 (2018)

65. K.J. May, C.E. Carlton, K.A. Stoerzinger, M. Risch, J. Suntivich, Y.L. Lee, A. Grimaud, Y. Shao-Horn, Influence of oxygen evolution during water oxidation on the surface of perovskite oxide catalysts. J. Phys. Chem. Lett. 3, 3264-3270 (2012)

66. B. Han, M. Risch, Y.L. Lee, C. Ling, H. Jia, Y. Shao-Horn, Activity and stability trends of perovskite oxides for oxygen evolution catalysis at neutral $\mathrm{pH}$. Phys. Chem. Chem. Phys. 17, 22576-22580 (2015)

67. A. Grimaud, K.J. May, C.E. Carlton, Y.L. Lee, M. Risch, W.T. Hong, J. Zhou, Y. Shao-Horn, Double perovskites as a family of highly active catalysts for oxygen evolution in alkaline solution. Nat. Commun. 4, 1-7 (2013)

68. D. Zu, H. Wang, S. Lin, G. Ou, H. Wei, S. Sun, H. Wu, Oxygendeficient metal oxides: synthesis routes and applications in energy and environment. Nano Res. 12, 2150-2163 (2019)

69. X.Y. Zhang, X.Q. Liu, Y.X. Zeng, Y.X. Tong, X.H. Lu, Oxygen defects in promoting the electrochemical performance of metal oxides for supercapacitors: recent advances and challenges. Small Methods 4, 1900823 (2020)

70. Y. Zhu, X. Liu, S. Jin, H. Chen, W. Lee, M. Liu, Y. Chen, Anionic defect engineering of transition metal oxides for oxygen reduction and evolution reactions. J. Mater. Chem. A 7, 5875-5897 (2019)

71. H. Wang, M. Zhou, P. Choudhury, H. Luo, Perovskite Oxides as Bifunctional Oxygen Electrocatalysts for Oxygen Evolution/ Reduction Reactions - A Mini Review. Applied Materials Today 16, 56-71 (2019)

72. A. Sarkar, G.G. Khan, The formation and detection techniques of oxygen vacancies in titanium oxide-based nanostructures. Nanoscale 11, 3414-3444 (2019)

73. Z. Shao, W. Zhou, Z. Zhu, Advanced synthesis of materials for intermediate-temperature solid oxide fuel cells. Prog. Mater. Sci. 57, 804-874 (2012)

74. X. Yao, J. Liu, W. Wang, F. Lu, W. Wang, Origin of OER catalytic activity difference of oxygen-deficient perovskites A2Mn2O5 (A = Ca, Sr): a theoretical study. J. Chem. Phys. 146, 224703-224703 (2017)

75. X. Rui, J. Walter, C. Leighton, R. Klie, Atomic-resolution study of oxygen vacancy ordering in Lao.5Sro.5CoO3-s thin films on 
SrTiO3 during in situ cooling experiments. Microsc. Microanal. 24, 84-85 (2018)

76. N. Biškup, J. Salafranca, V. Mehta, M.P. Oxley, Y. Suzuki, S.J. Pennycook, S.T. Pantelides, M. Varela, Insulating ferromagnetic LaCoO $3-\delta$ films: a phase induced by ordering of oxygen vacancies. Phys. Rev. Lett. 112, 087202 (2014)

77. R.K. Bera, H. Park, R. Ryoo, Co3O4 nanosheets on zeolitetemplated carbon as an efficient oxygen electrocatalyst for a zinc-air battery. J. Mater. Chem. A 7, 9988-9996 (2019)

78. X. Liu, L. Zhang, Y. Zheng, Z. Guo, Y. Zhu, H. Chen, F. Li, P. Liu, B. Yu, X. Wang, J. Liu, Y. Chen, M. Liu, Uncovering the effect of lattice strain and oxygen deficiency on electrocatalytic activity of perovskite cobaltite thin films. Adv Sci 6, 1801898 1801898 (2019)

79. W. Yang, H. Zhang, C. Sun, L. Liu, J.A. Alonso, M.T. FernándezDíaz, L. Chen, Insight into the structure and functional application of the $\mathrm{Sr} 0.95 \mathrm{Ce} 0.05 \mathrm{CoO} 3-\delta$ cathode for solid oxide fuel cells. Inorg. Chem. 54, 3477-3484 (2015)

80. Z. Li, L.E. Achenie, H. Xin, An adaptive machine learning strategy for accelerating discovery of perovskite electrocatalysts. ACS Catal. 10, 4377-4384 (2020)

81. J. Wang, C.-W. Sun, Y.-D. Gong, H.-R. Zhang, J.A. Alonso, M.T. Fernández-Díaz, Z.-L. Wang, J.B. Goodenough, Imaging the diffusion pathway of $\mathrm{Al}+$ ion in NASICON-type (A10. 2Zr0. 8) 20/ $19 \mathrm{Nb}$ (PO4) 3 as electrolyte for rechargeable solid-state Al batteries. Chin Phys B 27, 128201 (2018)

82. N.-T. Suen, S.F. Hung, Q. Quan, N. Zhang, Y.J. Xu, H.M. Chen, Electrocatalysis for the oxygen evolution reaction: Recent development and future perspectives. Chem. Soc. Rev. 46, 337-365 (2017)

83. R.-A. Eichel, Structural and dynamic properties of oxygen vacancies in perovskite oxides - analysis of defect chemistry by modern multi-frequency and pulsed EPR techniques. Phys. Chem. Chem. Phys. 13, 368-384 (2011)

84. L. Forni, C. Oliva, T. Barzetti, E. Selli, A.M. Ezerets, A.V. Vishniakov, FT-IR and EPR spectroscopic analysis of La(1$\mathrm{x}) \mathrm{Ce}(\mathrm{x}) \mathrm{CoO} 3$ perovskite-like catalysts for $\mathrm{NO}$ reduction by $\mathrm{CO}$. Appl. Catal. B Environ. 13, 35-43 (1997)

85. B. Wang, M. Zhang, X. Cui, Z. Wang, M. Rager, Y. Yang, Z. Zou, Z.L. Wang, Z. Lin, Unconventional route to oxygen-vacancyenabled highly efficient electron extraction and transport in perovskite solar cells. Angew. Chem. 132, 1628-1635 (2020)

86. L. Gui, Z. Wang, K. Zhang, B. He, Y. Liu, W. Zhou, J. Xu, Q. Wang, L. Zhao, Oxygen vacancies-rich Ce0.9Gd0.1O2- $\delta$ decorated Pr0.5Ba0.5CoO3- $\delta$ bifunctional catalyst for efficient and longlasting rechargeable Zn-air batteries. Appl. Catal. B Environ. 266, 118656-118656 (2020)

87. A. Anshul, M. Kumar, A. Raj, Raman and photoluminescence spectral studies in double perovskite epitaxial Nd2CoMnO6 thin films deposited by pulse laser deposition. Optik 212, 164749 164749 (2020)

88. A. Raj, M. Kumar, D. Mishra, A. Anshul, Raman and photoluminescence spectral studies in epitaxial Bi2NiMnO6 double perovskite thin-film samples. Opt. Mater. 101, 109773109773 (2020)

89. A.N. Ulyanov, A.V. Sidorov, N.E. Pismenova, E.A. Goodilin, S.V. Savilov, Self-doped La1-xMnO3 $+\delta$ perovskites: electron state hybridization and Raman modes. Solid State Sci. 94, 41-44 (2019)

90. P. Selvarajan, G. Chandra, S. Bhattacharya, S. Sil, A. Vinu, S. Umapathy, Potential of Raman spectroscopy towards understanding structures of carbon-based materials and perovskites. Emergent Mater 2, 417-439 (2019)

91. M.F. Mohamad Noh, N.A. Arzaee, J. Safaei, N.A. Mohamed, H.P. Kim, A.R. Mohd Yusoff, J. Jang, M.A. Mat Teridi, Eliminating oxygen vacancies in $\mathrm{SnO} 2$ films via aerosol-assisted chemical vapour deposition for perovskite solar cells and photoelectrochemical cells. J. Alloys Compd. 773, 997-1008 (2019)

92. S. Peng, F. Gong, L. Li, D. Yu, D. Ji, T. Zhang, Z. Hu, Z. Zhang, S. Chou, Y. Du, S. Ramakrishna, Necklace-like multishelled hollow spinel oxides with oxygen vacancies for efficient water electrolysis. J. Am. Chem. Soc. 140, 13644-13653 (2018)

93. C. Bloed, J. Vuong, A. Enriquez, S. Raghavan, I. Tran, S. Derakhshan, H. Tavassol, Oxygen vacancy and chemical ordering control oxygen evolution activity of $\mathrm{Sr} 2-\mathrm{xCaxFe} 2 \mathrm{O} 6-\delta$ perovskites. ACS Appl Energy Mater 2, 6140-6145 (2019)

94. A. Grimaud, O. Diaz-Morales, B. Han, W.T. Hong, Y.L. Lee, L. Giordano, K.A. Stoerzinger, M.T.M. Koper, Y. Shao-Horn, Activating lattice oxygen redox reactions in metal oxides to catalyse oxygen evolution. Nat. Chem. 9, 457-465 (2017)

95. I. Yamada, T. Odake, K. Asai, K. Oka, S. Kawaguchi, K. Wada, S. Yagi, High-pressure synthesis of highly oxidized $\mathrm{Ba} 0.5 \mathrm{Sr} 0.5 \mathrm{Co} 0.8 \mathrm{Fe} 0.2 \mathrm{O} 3-\delta$ cubic perovskite. Mater. Chemi. Front. 3, 1209-1217 (2019)

96. H. Lee, O. Gwon, K. Choi, L. Zhang, J. Zhou, J. Park, J.W. Yoo, J.Q. Wang, J.H. Lee, G. Kim, Enhancing bifunctional electrocatalytic activities via metal d-band center lift induced by oxygen vacancy on the subsurface of perovskites. ACS Catal. 10, 4664 $4670(2020)$

97. M. Imada, A. Fujimori, Y. Tokura, Metal-insulator transitions. Rev. Mod. Phys. 70, 1039-1263 (1998)

98. F.H.B. Lima, J. Zhang, M.H. Shao, K. Sasaki, M.B. Vukmirovic, E.A. Ticianelli, R.R. Adzic, Catalytic activity-d-band center correlation for the $\mathrm{O} 2$ reduction reaction on platinum in alkaline solutions. J. Phys. Chem. C 111, 404-410 (2007)

99. J.K. Nørskov, J. Rossmeisl, A. Logadottir, L. Lindqvist, J.R. Kitchin, T. Bligaard, H. Jónsson, Origin of the overpotential for oxygen reduction at a fuel-cell cathode. J. Phys. Chem. B 108, 17886-17892 (2004)

100. J.R. Petrie, H. Jeen, S.C. Barron, T.L. Meyer, H.N. Lee, Enhancing perovskite electrocatalysis through strain tuning of the oxygen deficiency. J. Am. Chem. Soc. 138, 7252-7255 (2016)

101. B. Zhao, L. Zhang, D. Zhen, S. Yoo, Y. Ding, D. Chen, Y. Chen, Q. Zhang, B. Doyle, X. Xiong, M. Liu, A tailored double perovskite nanofiber catalyst enables ultrafast oxygen evolution. Nat. Commun. 8, 1-9 (2017)

102. X. Xu, Y. Pan, W. Zhou, Y. Chen, Z. Zhang, Z. Shao, Toward enhanced oxygen evolution on perovskite oxides synthesized from different approaches: a case study of $\mathrm{Ba} 0.5 \mathrm{Sr} 0.5 \mathrm{Co} 0.8 \mathrm{Fe} 0.2 \mathrm{O} 3$ $-\delta$. Electrochim. Acta 219, 553-559 (2016)

103. X. Cheng, E. Fabbri, M. Nachtegaal, I.E. Castelli, M. El Kazzi, R. Haumont, N. Marzari, T.J. Schmidt, Oxygen evolution reaction on La1-xSrxCoO3 perovskites: a combined experimental and theoretical study of their structural, electronic, and electrochemical properties. Chem. Mater. 27, 7662-7672 (2015)

104. W. Zhou, J. Sunarso, Enhancing Bi-functional electrocatalytic activity of perovskite by temperature shock: a case study of LaNiO3ס. J. Phys. Chem. Lett. 4, 2982-2988 (2013)

105. L.J. Sham, W. Kohn, One-particle properties of an inhomogeneous interacting electron gas. Phys. Rev. 145, 561-567 (1966)

106. I.C. Man, H.Y. Su, F. Calle-Vallejo, H.A. Hansen, J.I. Martínez, N.G. Inoglu, J. Kitchin, T.F. Jaramillo, J.K. Nørskov, J. Rossmeisl, Universality in oxygen evolution electrocatalysis on oxide surfaces. ChemCatChem 3, 1159-1165 (2011)

107. H. Bae, J. Hong, B. Singh, A.K. Srivastava, J.H. Joo, S.-J. Song, Investigations on defect equilibrium, thermodynamic quantities, and transport properties of $\mathrm{La} 0.5 \mathrm{Sr} 0.5 \mathrm{FeO} 3-\delta$. J. Electrochem. Soc. 166, F180-F189 (2019)

108. Y. Shin, K.Y. Doh, S.H. Kim, J.H. Lee, H. Bae, S.J. Song, D. Lee, Effect of oxygen vacancies on electrical conductivity of 
La0.5Sr0.5FeO3-: $\delta$ from first-principles calculations. J. Mater. Chem. A 8, 4784-4789 (2020)

109. Y. Zhang, W. Yang, Comment on "generalized gradient approximation made simple". Phys. Rev. Lett. 80, 890 (1998)

110. C. Adamo, V. Barone, Toward reliable density functional methods without adjustable parameters: the PBE0 model. J. Chem. Phys. 110, 6158-6170 (1999)

111. J. Heyd, G.E. Scuseria, M. Ernzerhof, Hybrid functionals based on a screened Coulomb potential. J. Chem. Phys. 118, 8207-8215 (2003)

112. F. Aryasetiawan, O. Gunnarsson, The GW method. Rep. Prog. Phys. 61, 237-237 (1998) 312

113. J. Du, T. Zhang, F. Cheng, W. Chu, Z. Wu, J. Chen, Nonstoichiometric perovskite CaMnO3- $\delta$ for oxygen electrocatalysis with high activity. Inorg. Chem. 53, 9106-9114 (2014)

114. S. Švarcová, K. Wiik, J. Tolchard, H.J.M. Bouwmeester, T. Grande, Structural instability of cubic perovskite BaxSr1-xCo1yFeyO3- $\delta$. Solid State Ionics 178, 1787-1791 (2008)

115. J. Kim, S. Choi, A. Jun, H.Y. Jeong, J. Shin, G. Kim, Chemically stable perovskites as cathode materials for solid oxide fuel cells: La-doped Ba0.5Sr0.5Co0.8Fe 0.2O3- $\delta$. ChemSusChem 7, 1669 $1675(2014)$

116. S. Hirai, K. Morita, K. Yasuoka, T. Shibuya, Y. Tojo, Y. Kamihara, A. Miura, H. Suzuki, T. Ohno, T. Matsuda, S. Yagi,
Oxygen vacancy-originated highly active electrocatalysts for the oxygen evolution reaction. J. Mater. Chem. A 6, 15102-15109 (2018)

117. C. Sun, J.A. Alonso, J. Bian, Recent advances in perovskite-type oxides for energy conversion and storage applications. Adv. Energy Mater., 2000459 (2020)

118. Y. Bu, S. Kim, O. Kwon, Q. Zhong, G. Kim, A composite catalyst based on perovskites for overall water splitting in alkaline conditions. ChemElectroChem 6, 1520-1524 (2019)

119. R. Tang, Y. Nie, J.K. Kawasaki, D.-Y. Kuo, G. Petretto, G. Hautier, G.-M. Rignanese, K.M. Shen, D.G. Schlom, J. Suntivich, Oxygen evolution reaction electrocatalysis on $\mathrm{SrIrO} 3$ grown using molecular beam epitaxy. J. Mater. Chem. A 4, 6831$6836(2016)$

120. S. Hirai, T. Ohno, R. Uemura, T. Maruyama, M. Furunaka, R. Fukunaga, W.-T. Chen, H. Suzuki, T. Matsuda, S. Yagi, Ca 1 $-\mathrm{xSrxRuO} 3$ perovskite at the metal-insulator boundary as a highly active oxygen evolution catalyst. J. Mater. Chem. A 7, 1538715394 (2019)

121. X. Wang, B. Xiao, Y. Li, Y. Tang, F. Liu, J. Chen, Y. Liu, Firstprinciples based machine learning study of oxygen evolution reactions of perovskite oxides using a surface center-environment feature model. Appl. Surf. Sci. 531, 147323 (2020) 